
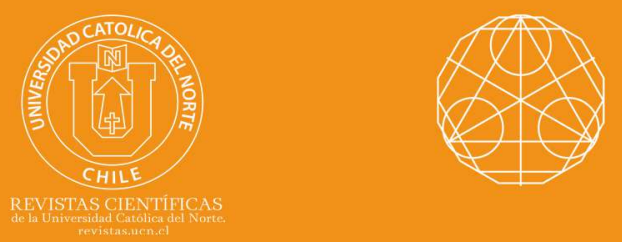

\title{
Other forms of continuity modulo an ideal
}

Néstor Raúl Pachón Rubiano ${ }^{10}$ orcid.org/0000-0002-7191-127X

${ }^{1}$ Escuela Colombiana de Ingeniería Julio Garavito, Dept of Mathematics, Bogotá, Colombia.

nestor.pachon@escuelaing.edu.co

\section{Abstract:}

The topic analized in this paper is the continuity modulo an ideal. We use the open-I sets to introduce new forms of weak continuity. Some basic properties of $C$-continuous and $D$-continuous functions will be investigated, as well as some applications related to compactness and separability. All the results obtained in this work constitute generalizations of well-known results of the general topology. We prove that these new concepts are independent of other forms of weak continuity, modulo an ideal, introduced by Abd El-Monsef, Özkurt, Çobankaya and Kaniewski.

Keywords: $\mathcal{J}$-continuous; $\mathcal{J}$-compact; $\mathcal{J}$-normal.

$\operatorname{MSC}(2020): 54 A 05,54 D 10,54 D 15$

Cite this article as (IEEE citation style):

N. R. Pachón Rubiano, "Other forms of continuity modulo an ideal", Proyecciones (Antofagasta, On line), vol. 39, no. 5, pp. 1221-1250, Oct. 2020, doi: 10.22199/issn.0717-6279-202005-0075.

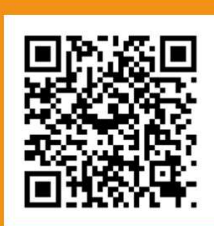

Article copyright: (C) 2020 Néstor Raúl Pachón Rubiano. This is an open access article distributed under the terms of the Creative Commons Licence, which permits unrestricted use and distribution provided the original author and source are credited. 


\section{Introduction and preliminaries}

Many concepts of the general topology have been extended to ideal topological spaces, and it is the case that the same concept has several extensions. The case of continuity is a good example of this fact. Authors such as Abd El-Monsef, Kaniewski, Özkurt and Çobankaya have studied different versions of weak continuity, modulo an ideal. In this paper we present two new forms of continuity for ideal topological spaces, which are not related to the versions presented by those authors.

An ideal $\mathcal{I}$ in a set $X$ is a subset of $\mathcal{P}(X)$, the power set of $X$, such that: ( $i$ ) if $A \subseteq B \subseteq X$ and $B \in \mathcal{I}$ then $A \in \mathcal{I}$, and (ii) if $A \in \mathcal{I}$ and $B \in \mathcal{I}$ then $A \cup B \in \mathcal{I}$.

Some simple and useful ideals in $X$ are: $(i) \mathcal{P}(A)$, where $A \subseteq X$, (ii) $\mathcal{I}_{f}(X)$, the ideal of all finite subsets of $X$ and $(i i i) \mathcal{I}_{c}(X)$, the ideal of all countable subsets of $X$. If $\mathcal{I}$ is an ideal in $X$ and if $f: X \rightarrow Y$ is a function, then the set $f(\mathcal{I})=\{f(I): I \in \mathcal{I}\}$ is an ideal in $Y$ [7]. Furthermore, if $\mathcal{J}$ is an ideal in $Y$ and if $f: X \rightarrow Y$ is an one-one function, then the set $f^{-1}(\mathcal{J})=\left\{f^{-1}(J): J \in \mathcal{J}\right\}$ is an ideal in $X[7]$. An ideal $\mathcal{I}$ in $X$ is said to be admissible if $\{x\} \in \mathcal{I}$, for each $x \in X$.

If $(X, \tau)$ is a topological space and $\mathcal{I}$ is an ideal in $X$, then $(X, \tau, \mathcal{I})$ is called an ideal space. If $(X, \tau)$ is a topological space and $A \subseteq X$ then the closure and the interior of $A$ are denoted by $\bar{A}$ (or $a d h_{\tau}(A)$, or $a d h(A)$ ) and $\AA$ (or $\operatorname{int}_{\tau}(A)$, or $\operatorname{int}(A)$ ), respectively. The frontier of $A$ is denoted by $\operatorname{Fr}(A)$.

Given an ideal space $(X, \tau, \mathcal{I})$ and a set $A \subseteq X$, we denote by $A^{*}(\mathcal{I})=$ $\{x \in X: U \cap A \notin \mathcal{I}$, for every $U \in \tau$ with $x \in U\}$, written simply as $A^{*}$ when there is no chance for confusion. It is clear that $A^{*} \subseteq \bar{A}$. A Kuratowski closure operator for a topology $\tau^{*}(\mathcal{I})$, finer than $\tau$, is defined by $C l^{*}(A)=A \cup A^{*}$, for all $A \subseteq X$. When there is no chance for confusion $\tau^{*}(\mathcal{I})$ is denoted by $\tau^{*}$. The topology $\tau^{*}$ has as a base $\beta(\tau, \mathcal{I})=$ $\{V \backslash I: V \in \tau$ and $I \in \mathcal{I}\}$ [5]. In 1990, D. Jancovic and T. R. Hamlett introduced the notion of $\mathcal{I}$-open sets. If $(X, \tau, \mathcal{I})$ is an ideal space and $A \subseteq X$, $A$ is said to be $\mathcal{I}$-open [4] if $A \subseteq \operatorname{int}\left(A^{*}\right) . A$ is said to be $\mathcal{I}$-closed if $X \backslash A$ is $\mathcal{I}$-open. On the other hand, $A$ is said to be closed- $\mathcal{I}[10]$ if $\bar{A} \backslash A \in \mathcal{I}$. If $X \backslash A$ is closed- $\mathcal{I}$ then $A$ is defined to be open- $\mathcal{I}$. It is immediate that $A$ is open- $\mathcal{I}$ if and only if $A \backslash \AA \in \mathcal{I}$.

If $\mathcal{I}$ is an ideal in $X$, let $\mathcal{I}^{\otimes}=\mathcal{P}\left(\bigcup_{I \in \mathcal{I}} I\right)$ be. If $(X, \tau, \mathcal{I})$ is an ideal space, the ideal $\overline{\mathcal{I}}[10]$ is the set $\{A \subseteq X: A \subseteq \bar{I}$, for some $I \in \mathcal{I}\}$. 
It is observed that if $\left\{F_{\alpha}\right\}_{\alpha \in \Lambda}$ is a locally finite collection of closed- $\mathcal{I}$ sets, then $\bigcup_{\alpha \in \Lambda} F_{\alpha}$ is closed-I $\mathcal{I}^{\otimes}$. In fact, since $\left\{F_{\alpha}\right\}_{\alpha \in \Lambda}$ is locally finite we have that $\overline{\bigcup_{\alpha \in \Lambda} F_{\alpha}}=\bigcup_{\alpha \in \Lambda} \overline{F_{\alpha}}$, and so $\overline{\bigcup_{\alpha \in \Lambda} F_{\alpha}} \backslash \bigcup_{\alpha \in \Lambda} F_{\alpha}=\left(\bigcup_{\alpha \in \Lambda} \overline{F_{\alpha}}\right) \backslash\left(\bigcup_{\alpha \in \Lambda} F_{\alpha}\right) \subseteq$ $\bigcup_{\alpha \in \Lambda}\left(\overline{F_{\alpha}} \backslash F_{\alpha}\right) \in \mathcal{I}^{\otimes}$.

\section{2. $\mathcal{C}$-continuous functions}

In 2014 Özkurt defines the $\mathcal{J}$-continuous functions, as an extension of the continuous functions to the ideal topological spaces. Years later, in 2017, Çobankaya et al. defines the $\mathcal{J}_{w}$-continuous functions, as a generalization of the concept studied by Özkurt. In both cases the considered ideal is defined in the codomain of the function. Under the same hypothesis, in this section we define a new and natural extension of the continuous functions to the ideal topological spaces, which turns out to be independent of the concepts given by these two authors. Various properties and examples will be presented.

Recall that if $(X, \tau)$ and $(Y, \beta)$ are topological spaces and $\mathcal{J}$ is an ideal in $Y$, a function $f:(X, \tau) \rightarrow(Y, \beta, \mathcal{J})$ is said to be $\mathcal{J}$-continuous [8] if for each $x \in X$ and each $V \in \beta$, if $f(x) \in V$ then there exists $U \in \tau$ such that $x \in U$ and $f(U) \backslash V \in \mathcal{J}$. A function $f:(X, \tau) \rightarrow(Y, \beta, \mathcal{J})$ is said to be $\mathcal{J}_{w}$-continuous [2] if for each $x \in X$ and each $V \in \beta$, if $f(x) \in V$ then there is a $U \in \tau$ such that $x \in U$ and $f(U) \backslash \bar{V} \in \mathcal{J}$. Evidently $\mathcal{J}$-continuous $\rightarrow \mathcal{J}_{w}$-continuous.

Definition 2.1 If $\mathcal{J}$ is an ideal in $Y$, a function $f:(X, \tau) \rightarrow(Y, \beta, \mathcal{J})$ is said to be $\mathcal{C}$-continuous if, for each $A \subseteq X, f(\bar{A}) \backslash \overline{f(A)} \in \mathcal{J}$.

It is clear that if $f$ is continuous then $f$ is $\mathcal{C}$-continuous, and that $f$ : $(X, \tau) \rightarrow(Y, \beta)$ is continuous if and only if $f:(X, \tau) \rightarrow(Y, \beta,\{\emptyset\})$ is $\mathcal{C}$ continuous. It is easy to see that if $g:(X, \tau) \rightarrow(Y, \beta)$ is continuous and $h:(Y, \beta) \rightarrow(Z, \gamma, \mathcal{J})$ is $\mathcal{C}$-continuous, then $h \circ g$ is $\mathcal{C}$-continuous. Also it is immediate that $f:(X, \tau) \rightarrow\left(Y, \beta, \mathcal{J}_{\alpha}\right)$ is $\mathcal{C}$-continuous, for each $\alpha \in \Lambda$, if and only if $f:(X, \tau) \rightarrow(Y, \beta, \mathcal{J})$ is $\mathcal{C}$-continuous, where $\mathcal{J}=\bigcap_{\alpha \in \Lambda} \mathcal{J}_{\alpha}$.

Example 2.2 (1) If $\mathcal{U}$ is the usual topology in $\mathbf{R}$, the identity function $f$ : $(\mathbf{R}, \mathcal{U}) \rightarrow(\mathbf{R}, \mathcal{P}(\mathbf{R}), \mathcal{J}=\mathcal{P}(\mathbf{R}))$ is $\mathcal{C}$-continuous, but $f$ is not continuous.

(2) If $\beta=\{\emptyset, \mathbf{R},\{0\}\}$ and $\mathcal{J}=\mathcal{P}(\{-1,0,1\})$, the function $f:(\mathbf{R}, \mathcal{U}) \rightarrow$ $(\mathbf{R}, \beta, \mathcal{J})$ defined by 


$$
f(x)=\left\{\begin{array}{c}
1, \text { if } x>0 \\
0, \text { if } x=0 \\
-1, \text { if } x<0
\end{array}\right.
$$

is $\mathcal{C}$-continuous but $f$ is not continuous.

(3) The function $f:(\mathbf{R}, \mathcal{U}) \rightarrow(\mathbf{R}, \mathcal{U}, \mathcal{J}=\mathcal{P}(\{0\}))$ defined by $f(x)=[x]$, where $[x]$ denotes the integer part of $x$, is not $\mathcal{C}$-continuous, because if $A=\left\{1-1 / n: n \in \mathbf{Z}^{+}\right\}$then $f(\bar{A}) \backslash \overline{f(A)}=\{1\} \notin \mathcal{J}$.

Next we present a characterization of the $\mathcal{C}$-continuity in terms of the interior operator.

Theorem 2.3 The function $f:(X, \tau) \rightarrow(Y, \beta, \mathcal{J})$ is $\mathcal{C}$-continuous if and only if, for each $B \subseteq Y$, there exists $J \in \mathcal{J}$ such that $f^{-1}\left(B^{\circ} \backslash J\right) \subseteq$ int $\left(f^{-1}(B)\right)$ or, equivalently, $f^{-1}(\stackrel{\circ}{\mathrm{B}}) \subseteq \operatorname{int}\left(f^{-1}(B)\right) \cup f^{-1}(J)$. Then $f$ is $\mathcal{C}$-continuous if and only if, for all $B \subseteq Y, f\left[f^{-1}\left(B^{\circ}\right) \backslash\right.$ int $\left.\left(f^{-1}(B)\right)\right] \in$ $\mathcal{J}$.

Proof. $(\rightarrow)$ Suppose that $B \subseteq Y$. There exists $J \in \mathcal{J}$ with $f\left[\overline{f^{-1}(Y \backslash B)}\right] \subseteq$ $\overline{f\left[f^{-1}(Y \backslash B)\right]} \cup J \subseteq \overline{(Y \backslash B)} \cup J=\left[Y \backslash B^{\circ}\right] \cup J$. Then $f\left[X \backslash\right.$ int $\left.\left(f^{-1}(B)\right)\right] \subseteq$ $\left(Y \backslash B^{\circ}\right) \cup J$, and so $X \backslash \operatorname{int}\left(f^{-1}(B)\right) \subseteq f^{-1}\left(Y \backslash B^{\circ}\right) \cup f^{-1}(J)$.

Hence $f^{-1}\left(B^{\circ} \backslash J\right) \subseteq$ int $\left(f^{-1}(B)\right)$.

$(\leftarrow)$ If $A \subseteq X$ then there is a $J \in \mathcal{J}$ with $f^{-1}[\operatorname{int}(Y \backslash f(A)) \backslash J] \subseteq$ $\operatorname{int}\left[f^{-1}(Y \backslash f(A))\right]$. Thus $f^{-1}[(Y \backslash \overline{f(A)}) \backslash J] \subseteq X \backslash \overline{f^{-1}(f(A))} \subseteq X \backslash \bar{A}$, and so $\bar{A} \subseteq f^{-1}[\overline{f(A)} \cup J]$ or, equivalently, $f(\bar{A}) \subseteq \overline{f(A)} \cup J$, and this implies that $f(\bar{A}) \backslash \overline{f(A)} \in \mathcal{J}$.

Corollary 2.4 (1) The following propositions are equivalents:

(a) The function $f:(X, \tau) \rightarrow(Y, \beta, \mathcal{J})$ is $\mathcal{C}$-continuous. (b) For each $V \in \beta$, there exists $J \in \mathcal{J}$ such that $f^{-1}(V \backslash J) \subseteq$ int $\left(f^{-1}(V)\right)$, this is $f\left[f^{-1}(V) \backslash \operatorname{int}\left(f^{-1}(V)\right)\right] \in \mathcal{J}$. (c) For each $B \subseteq Y$, there is a $J \in \mathcal{J}$ such that $\overline{f^{-1}(B)} \subseteq f^{-1}(\bar{B} \cup J)$ or, equivalently, $f\left[\overline{f^{-1}(B)} \backslash f^{-1}(\bar{B})\right] \in \mathcal{J}$. (d) For each closed $F \subseteq Y, f\left[\overline{f^{-1}(F)} \backslash f^{-1}(F)\right] \in \mathcal{J}$. (2) If $f:(X, \tau) \rightarrow$ $(Y, \beta, \mathcal{J})$ is one-one, then the following statements are equivalents: (a) $f$ is $\mathcal{C}$-continuous. (b) For each $B \subseteq Y, \overline{f^{-1}(B)} \backslash f^{-1}(\bar{B}) \in f^{-1}(\mathcal{J})$. (c) For each closed set $B \subseteq Y, f^{-1}(B)$ is closed- $f^{-1}(\mathcal{J})$. (d) For each $V \subseteq Y, f^{-1}\left(V^{\circ}\right) \backslash i n t\left[f^{-1}(V)\right] \in f^{-1}(\mathcal{J})$. (e) For each $V \in \beta, f^{-1}(V)$ 
is open- $f^{-1}(\mathcal{J})$. (3) If $\mathcal{I}$ is an ideal in $X$ and if $f: X \rightarrow Y$ is an one-one function, then $f:(X, \tau) \rightarrow(Y, \beta, f(\mathcal{I}))$ is $\mathcal{C}$-continuous if and only if $f^{-1}(V)$ is open- $\mathcal{I}$, for each $V \in \beta$.

Now we present a characterization of the $\mathcal{C}$-continuous functions, in terms of the frontier operator.

Theorem 2.5 The function $f:(X, \tau) \rightarrow(Y, \beta, \mathcal{J})$ is $\mathcal{C}$-continuous if and only if, for each $B \subseteq Y$, there exists $J \in \mathcal{J}$ such that $\operatorname{Fr}\left[f^{-1}(B)\right] \subseteq$ $f^{-1}(\operatorname{Fr}(B) \cup J)$ or, equivalently, $f\left[F r\left(f^{-1}(B)\right) \backslash f^{-1}(F r(B))\right] \in \mathcal{J}$.

Proof. $(\rightarrow)$ If $B \subseteq Y$, there is a $\left\{J_{1}, J_{2}\right\} \subseteq \mathcal{J}$ such that $\overline{f^{-1}(B)} \subseteq$ $f^{-1}\left(\bar{B} \cup J_{1}\right)$ and $\overline{f^{-1}(Y \backslash B)} \subseteq f^{-1}\left(\overline{Y \backslash B} \cup J_{2}\right)$.

So $F r\left(f^{-1}(B)\right)=\overline{f^{-1}(B)} \cap \overline{X \backslash f^{-1}(B)} \subseteq f^{-1}\left[\left(\bar{B} \cup J_{1}\right) \cap\left(\overline{Y \backslash B} \cup J_{2}\right)\right]$ $=f^{-1}\left[F r(B) \cup\left(\bar{B} \cap J_{2}\right) \cup\left(J_{1} \cap \overline{Y \backslash B}\right) \cup\left(J_{1} \cap J_{2}\right)\right]=f^{-1}[F r(B) \cup J]$, where $J=\left(\bar{B} \cap J_{2}\right) \cup\left(J_{1} \cap \overline{Y \backslash B}\right) \cup\left(J_{1} \cap J_{2}\right) \in \mathcal{J}$.

$(\leftarrow)$ Suppose that $F \subseteq Y$ is closed. There is a $J \in \mathcal{J}$ such that $\operatorname{Fr}\left[f^{-1}(F)\right] \subseteq f^{-1}(F r(F) \cup J) \subseteq f^{-1}(F \cup J)=f^{-1}(F) \cup f^{-1}(J)$.

Hence

$\overline{f^{-1}(F)} \backslash \operatorname{int}\left(f^{-1}(F)\right) \subseteq f^{-1}(F) \cup f^{-1}(J)$, and so $\overline{f^{-1}(F)} \subseteq \operatorname{int}\left(f^{-1}(F)\right) \cup$ $f^{-1}(F) \cup f^{-1}(J)=f^{-1}(F) \cup f^{-1}(J)$. Corollary 2.4 implies that $f$ is $\mathcal{C}$ continuous.

The following characterization of $\mathcal{C}$-continuous (and one-one) functions is expressed completely in terms of closed- $\mathcal{I}$ sets.

Theorem 2.6 If $f:(X, \tau) \rightarrow(Y, \beta, \mathcal{J})$ is one-one, then $f$ is $\mathcal{C}$ continuous if and only if, for each closed- $\mathcal{J}$ set $B \subseteq Y$, it is true that $f^{-1}(B)$ is closed- $f^{-1}(\mathcal{J})$.

Proof. $(\rightarrow)$ If $B \subseteq Y$ is closed- $\mathcal{J}$, we have that $\bar{B} \backslash B \in \mathcal{J}$, and so $f^{-1}(\bar{B}) \backslash f^{-1}(B)=f^{-1}(\bar{B} \backslash B) \in f^{-1}(\mathcal{J})$. In addition, $\overline{f^{-1}(B)} \backslash f^{-1}(\bar{B}) \in$ $f^{-1}(\mathcal{J})$, by Corollary 2.4. This implies that $\overline{f^{-1}(B)} \backslash f^{-1}(B) \in f^{-1}(\mathcal{J})$. $(\leftarrow)$ It is a consequence of Corollary 2.4, given that if $F \subseteq Y$ is closed then $F$ is closed- $\mathcal{J}$.

Corollary 2.7 If $\mathcal{I}$ is an ideal in $X$ and if $f: X \rightarrow Y$ is an one-one function, then $f:(X, \tau) \rightarrow(Y, \beta, f(\mathcal{I}))$ is $\mathcal{C}$-continuous if and only if $f^{-1}(V)$ is open- $\mathcal{I}$, for each open- $f(\mathcal{I})$ set $V \subseteq Y$. 
Theorem 2.8 Suppose that $\mathcal{I}$ is an ideal in $X$ and $f: X \rightarrow Y$ is an oneone function. If $\mathcal{J}=\left\{B \subseteq Y: f^{-1}(B) \in \mathcal{I}\right\}$ then $f:(X, \tau) \rightarrow(Y, \beta, \mathcal{J})$ is $\mathcal{C}$-continuous if and only if $f^{-1}(B)$ is closed- $\mathcal{I}$, for each closed- $\mathcal{J}$ set $B \subseteq Y$.

Proof. $(\rightarrow)$ If $B \subseteq Y$ is closed- $\mathcal{J}$ then $\bar{B} \backslash B \in \mathcal{J}$, and so $f^{-1}(\bar{B}) \backslash f^{-1}(B) \in$ $\mathcal{I}$. Since $f$ is $\mathcal{C}$-continuous we have that $\overline{f^{-1}(B)} \backslash f^{-1}(\bar{B}) \in f^{-1}(\mathcal{J}) \subseteq \mathcal{I}$. Given that $\overline{f^{-1}(B)} \backslash f^{-1}(B) \subseteq\left[f^{-1}(\bar{B}) \backslash f^{-1}(B)\right] \cup\left[\overline{f^{-1}(B)} \backslash f^{-1}(\bar{B})\right]$, then $\overline{f^{-1}(B)} \backslash f^{-1}(B) \in \mathcal{I}$.

$(\leftarrow)$ If $B \subseteq Y$ then $\overline{f^{-1}(\bar{B})} \backslash f^{-1}(\bar{B}) \in \mathcal{I}$, since $\bar{B}$ is closed- $\mathcal{J}$, and so $\overline{f^{-1}(B)} \backslash f^{-1}(\bar{B}) \in \mathcal{I}$. There exists $I \in \mathcal{I}$ with $\overline{f^{-1}(B)} \backslash f^{-1}(\bar{B})=I=$ $f^{-1}(f(I))$. Moreover $f(I) \in \mathcal{J}$.

The following two properties of the $\mathcal{C}$-continuity are related with restrictions of functions.

Theorem 2.9 If $f:(X, \tau) \rightarrow(Y, \beta, \mathcal{J})$ is $\mathcal{C}$-continuous and $A \subseteq X$, then the function $f_{A}:\left(A, \tau_{A}\right) \rightarrow(Y, \beta, \mathcal{J})$ is $\mathcal{C}$-continuous, where $f_{A}$ is the restriction of $f$ to $A$.

Proof. If $V \in \beta$, there is a $J \in \mathcal{J}$ such that $f^{-1}(V) \subseteq$ int $\left(f^{-1}(V)\right) \cup$ $f^{-1}(J)$. Thus $f_{A}^{-1}(V)=A \cap f^{-1}(V) \subseteq\left[A \cap i n t\left(f^{-1}(V)\right)\right] \cup\left[A \cap f^{-1}(J)\right]=$ $\left[A \cap \operatorname{int}\left(f^{-1}(V)\right)\right] \cup f_{A}^{-1}(J)$. But $A \cap i n t\left(f^{-1}(V)\right) \subseteq i n t_{\tau_{A}}\left[A \cap f^{-1}(V)\right]=$ $\operatorname{int}_{\tau_{A}}\left(f_{A}^{-1}(V)\right)$. Hence $f_{A}^{-1}(V) \subseteq i n t_{\tau_{A}}\left(f_{A}^{-1}(V)\right) \cup f_{A}^{-1}(J)$.

Theorem 2.10 Suppose that $(X, \tau)$ is a topological space and that $X=$ $U \cup V$, where $\{U, V\} \subseteq \tau$. If $f:(X, \tau) \rightarrow(Y, \beta, \mathcal{J})$ is a function such that $f_{U}:\left(U, \tau_{U}\right) \rightarrow(Y, \beta, \mathcal{J})$ and $f_{V}:\left(V, \tau_{V}\right) \rightarrow(Y, \beta, \mathcal{J})$ are $\mathcal{C}$-continuous, then $f$ is $\mathcal{C}$-continuous.

Proof. If $W \in \beta$, there is a $\left\{J_{1}, J_{2}\right\} \subseteq \mathcal{J}$ such that $f_{U}^{-1}(W) \subseteq$ int $_{\tau_{U}}\left(f_{U}^{-1}(W)\right) \cup f_{U}^{-1}\left(J_{1}\right)$ and $f_{V}^{-1}(W) \subseteq \operatorname{int}_{\tau_{V}}\left(f_{V}^{-1}(W)\right) \cup f_{V}^{-1}\left(J_{2}\right)$. Then $f^{-1}(W)=f_{U}^{-1}(W) \cup f_{V}^{-1}(W) \subseteq i n t_{\tau_{U}}\left(f_{U}^{-1}(W)\right) \cup i n t_{\tau_{V}}\left(f_{V}^{-1}(W)\right) \cup$ $f_{U}^{-1}\left(J_{1}\right) \cup f_{V}^{-1}\left(J_{2}\right)$. Given that $U$ and $V$ are open, int $\tau_{\tau_{U}}\left(f_{U}^{-1}(W)\right)=U \cap$ $\operatorname{int}\left(f^{-1}(W)\right)$ and $\operatorname{int}_{\tau_{V}}\left(f_{V}^{-1}(W)\right)=V \cap \operatorname{int}\left(f^{-1}(W)\right)$. Thus $f^{-1}(W) \subseteq$ $\left[U \cap \operatorname{int}\left(f^{-1}(W)\right)\right]$ $\cup\left[V \cap \operatorname{int}\left(f^{-1}(W)\right)\right] \cup f^{-1}\left(J_{1} \cup J_{2}\right)=\operatorname{int}\left(f^{-1}(W)\right) \cup f^{-1}\left(J_{1} \cup J_{2}\right)$. 
Recall that if $A \subseteq X$, the characteristic function, associated with $A$, is the function $\chi_{A}: X \rightarrow\{0,1\}$ defined by: $\chi_{A}(x)=1$, if $x \in A$, and $\chi_{A}(x)=0$, if $x \notin A$.

Theorem 2.11 If $(X, \tau)$ is a topological space, $A \subseteq X, \mathcal{J}_{1}=\{\emptyset,\{0\}\}$ and $\mathcal{J}_{2}=\{\emptyset,\{1\}\}$ then:

(1) The function $\chi_{A}:(X, \tau) \rightarrow\left(\{0,1\}, \mathcal{P}(\{0,1\}), \mathcal{J}_{1}\right)$ is $\mathcal{C}$-continuous if and only if $A$ is open. (2) The function $\chi_{A}:(X, \tau) \rightarrow\left(\{0,1\}, \mathcal{P}(\{0,1\}), \mathcal{J}_{2}\right)$ is $\mathcal{C}$-continuous if and only if $A$ is closed.

Proof. $(1)(\rightarrow)$ There is a $J \in \mathcal{J}_{1}$ such that $A=\chi_{A}^{-1}(\{1\}) \subseteq \chi_{A}^{-1}(J) \cup$ $\operatorname{int}\left(\chi_{A}^{-1}(\{1\})\right)=A^{\circ} \cup \chi_{A}^{-1}(J) \subseteq A^{\circ} \cup(X \backslash A)$. Hence $A \subseteq A^{\circ}$, and then $A$ is open. $(\leftarrow)$ It is sufficient to note that: (a) $\chi_{A}^{-1}(\{0\}) \subseteq \chi_{A}^{-1}(\{0\}) \cup$ $\operatorname{int}\left(\chi_{A}^{-1}(\{0\})\right)$, and (b) $\chi_{A}^{-1}(\{1\})=A \subseteq \chi_{A}^{-1}(J) \cup A=\chi_{A}^{-1}(J) \cup \operatorname{int}\left(\chi_{A}^{-1}(\{1\})\right)$, for all $J \in \mathcal{J}_{1}$.

(2) It is similar to (1).

Theorem 2.12 Let $(Y, \beta)$ be a topological space, $\mathcal{B}$ a base for $\beta$ and $\mathcal{J}$ an ideal in $Y$. The function $f:(X, \tau) \rightarrow\left(Y, \beta, \mathcal{J}^{\otimes}\right)$ is $\mathcal{C}$-continuous if and only if, for each $B \in \mathcal{B}$, there exists $J \in \mathcal{J}^{\otimes}$ such that $f^{-1}(B) \subseteq$ int $\left(f^{-1}(B)\right) \cup f^{-1}(J)$ or, equivalently, $f\left[f^{-1}(B) \backslash \operatorname{int}\left(f^{-1}(B)\right)\right] \in \mathcal{J}^{\otimes}$.

Proof. $(\rightarrow)$ It is clear.

$(\leftarrow)$ If $V \in \beta$ then there exists $\left\{B_{\alpha}: \alpha \in \Delta\right\} \subseteq \mathcal{B}$, such that $V=$ $\bigcup B_{\alpha}$. If $\alpha \in \Delta$, there is a $J_{\alpha} \in \mathcal{J}^{\otimes}$ with $f^{-1}\left(B_{\alpha}\right) \subseteq \operatorname{int}\left(f^{-1}\left(B_{\alpha}\right)\right) \cup$ $f^{-1}\left(J_{\alpha}\right)$. If $J=\bigcup_{\alpha \in \Delta} J_{\alpha}$ then $J \in \mathcal{J}^{\otimes}$.

Now, $f^{-1}(V)=\bigcup_{\alpha \in \Delta} f^{-1}\left(B_{\alpha}\right) \subseteq \bigcup_{\alpha \in \Delta}\left[\operatorname{int}\left(f^{-1}\left(B_{\alpha}\right)\right) \cup f^{-1}\left(J_{\alpha}\right)\right] \subseteq \operatorname{int}\left(f^{-1}(V)\right)$ $\cup f^{-1}(J)$.

Theorem 2.13 Let $(Y, \beta)$ be a topological space, $\mathcal{S}$ a sub-base for $\beta$ and $\mathcal{J}$ an ideal in $Y$. The function $f:(X, \tau) \rightarrow\left(Y, \beta, \mathcal{J}^{\otimes}\right)$ is $\mathcal{C}$ continuous if and only if for each $S \in \mathcal{S}$ there exists $J \in \mathcal{J}^{\otimes}$ such that $f^{-1}(S) \subseteq \operatorname{int}\left(f^{-1}(S)\right) \cup f^{-1}(J)$.

Proof. $(\rightarrow)$ It is clear.

$(\leftarrow)$ By Theorem 2.12, it is enough to see that if $\left\{S_{1}, S_{2}\right\} \subseteq \mathcal{S}$, there exists $J \in \mathcal{J}^{\otimes}$ such that $f^{-1}\left(S_{1} \cap S_{2}\right) \subseteq \operatorname{int}\left(f^{-1}\left(S_{1} \cap S_{2}\right)\right) \cup f^{-1}(J)$. If 
$\left\{S_{1}, S_{2}\right\} \subseteq \mathcal{S}$, there is a $\left\{J_{1}, J_{2}\right\} \subseteq \mathcal{J}^{\otimes}$ such that $f^{-1}\left(S_{i}\right) \subseteq \operatorname{int}\left(f^{-1}\left(S_{i}\right)\right) \cup$ $f^{-1}\left(J_{i}\right)$, for all $i \in\{1,2\}$.

Thus $f^{-1}\left(S_{1} \cap S_{2}\right)=f^{-1}\left(S_{1}\right) \cap f^{-1}\left(S_{2}\right) \subseteq\left[\operatorname{int}\left(f^{-1}\left(S_{1}\right)\right) \cup f^{-1}\left(J_{1}\right)\right] \cap$ $\left[\operatorname{int}\left(f^{-1}\left(S_{2}\right)\right) \cup f^{-1}\left(J_{2}\right)\right]=\left[\operatorname{int}\left(f^{-1}\left(S_{1}\right)\right) \cap \operatorname{int}\left(f^{-1}\left(S_{2}\right)\right)\right] \cup A \cup B \cup$ $f^{-1}\left(J_{3}\right)$, where $J_{3}=J_{1} \cap J_{2}, A=\operatorname{int}\left(f^{-1}\left(S_{1}\right)\right) \cap f^{-1}\left(J_{2}\right)$ and $B=$ $f^{-1}\left(J_{1}\right) \cap i n t\left(f^{-1}\left(S_{2}\right)\right)$. $\mathcal{J}^{\otimes}$.

Hence $f^{-1}\left(S_{1} \cap S_{2}\right) \subseteq \operatorname{int}\left[f^{-1}\left(S_{1} \cap S_{2}\right)\right] \cup f^{-1}\left(J_{2} \cup J_{1}\right)$, and $J_{2} \cup J_{1} \in$

Theorem 2.14 If $f:(X, \tau) \rightarrow(Y, \beta, \mathcal{J})$ and $g:(Y, \beta) \rightarrow(Z, \gamma, \mathcal{L})$ are $\mathcal{C}$ -continuous, and if $g(\mathcal{J}) \subseteq \mathcal{L}$ then $g \circ f$ is $\mathcal{C}$-continuous.

Proof. If $A \subseteq X$, there are $J \in \mathcal{J}$ and $L \in \mathcal{L}$ such that $f(\bar{A}) \subseteq$ $\overline{f(A)} \cup J$ and $g(\overline{f(A)}) \subseteq \overline{(g \circ f)(A)} \cup L$. Thus $(g \circ f)(\bar{A})=g(f(\bar{A})) \subseteq$ $g(\overline{f(A)}) \cup g(J) \subseteq \overline{(g \circ f)(A)} \cup L \cup g(J)$, with $L \cup g(J) \in \mathcal{L}$.

Corollary 2.15 If $f:(X, \tau) \rightarrow(Y, \beta, \mathcal{J})$ and $g:(Y, \beta) \rightarrow(Z, \gamma, g(\mathcal{J}))$ are $\mathcal{C}$-continuous, then $g \circ f$ is $\mathcal{C}$-continuous.

The example that follows shows us that, in general, the composition of $\mathcal{C}$-continuous functions is not $\mathcal{C}$-continuous.

Example 2.16 If $X=Y=Z=\{a, b, c\}, \tau=\{\emptyset, X,\{a\},\{a, b\},\{a, c\}\}$, $\beta=\{\emptyset, Y,\{b\},\{a, b\},\{b, c\}\}, \gamma=\{\emptyset, Z,\{c\},\{a, c\},\{b, c\}\}, \mathcal{L}=\{\emptyset,\{a\}\}$, $\mathcal{J}=\{\emptyset,\{a\},\{b\},\{a, b\}\}$, and if $f: X \rightarrow Y$ and $g: Y \rightarrow Z$ are defined by $f(a)=c, f(b)=a, f(c)=b, g(a)=a=g(c)$ and $g(b)=b$, it is easy to see that $f$ and $g$ are $\mathcal{C}$-continuous. However, since $(g \circ f)(\overline{\{a\}}) \backslash \overline{(g \circ f)(\{a\})}=$ $\{a, b\} \backslash\{a\}=\{b\} \notin \mathcal{L}$, we have that $g \circ f$ is not $\mathcal{C}$-continuous.

Theorem 2.17 If $f:(X, \tau) \rightarrow(Y, \beta)$ is a function, $\left\{X_{\alpha}\right\}_{\alpha \in \Lambda}$ is a locally finite collection of closed subsets of $X$ whose union is $X$, and if $\mathcal{J}$ is an ideal on $Y$, then $f:(X, \tau) \rightarrow\left(Y, \beta, \mathcal{J}^{\otimes}\right)$ is $\mathcal{C}$-continuous if and only if each restriction $f_{X_{\alpha}}:\left(X_{\alpha}, \tau_{\alpha}\right) \rightarrow\left(Y, \beta, \mathcal{J}^{\otimes}\right)$ is $\mathcal{C}$-continuous. Here $\tau_{\alpha}=\tau_{X_{\alpha}}$.

Proof. $(\leftarrow)$ For each $\alpha \in \Lambda$, we will denote the function $f_{X_{\alpha}}$ simply by $f_{\alpha}$. Suppose that $F \subseteq Y$ is closed. Since $f^{-1}(F)=\bigcup_{\alpha \in \Lambda} f_{\alpha}^{-1}(F)$ and $\left\{f_{\alpha}^{-1}(F)\right\}_{\alpha \in \Lambda}$ is locally finite, we have that $\overline{f^{-1}(F)}=\frac{\alpha \in \Lambda}{\bigcup_{\alpha \in \Lambda} f_{\alpha}^{-1}(F)}=$ 
$\bigcup_{\alpha \in \Lambda} \overline{f_{\alpha}^{-1}(F)}$. Now, for each $\alpha \in \Lambda$, there is a $J_{\alpha} \in \mathcal{J}^{\otimes}$ such that $a d h_{\tau_{\alpha}}\left(f_{\alpha}^{-1}(F)\right) \subseteq$ $f_{\alpha}^{-1}\left(F \cup J_{\alpha}\right)$. Given that each $X_{\alpha}$ is closed, $a d h_{\tau_{\alpha}}\left(f_{\alpha}^{-1}(F)\right)=\overline{f_{\alpha}^{-1}(F)}$.

Hence $\overline{f^{-1}(F)} \subseteq \bigcup_{\alpha \in \Lambda} f_{\alpha}^{-1}(F) \cup f^{-1}\left(\bigcup_{\alpha \in \Lambda} J_{\alpha}\right)=f^{-1}(F) \cup f^{-1}\left(\bigcup_{\alpha \in \Lambda} J_{\alpha}\right)=$ $f^{-1}\left(F \cup \bigcup_{\alpha \in \Lambda} J_{\alpha}\right)$, with $\bigcup_{\alpha \in \Lambda} J_{\alpha} \in \mathcal{J}^{\otimes}$.

$(\rightarrow)$ It is a consequence of Theorem 2.9.

Notation 2.18 If $\mathcal{I}$ is an ideal in $X$ and $\mathcal{J}$ is an ideal in $Y$, then $\mathcal{I} \otimes \mathcal{J}$ [10] is the set of all $D \subseteq X \times Y$ such that there exist $I \in \mathcal{I}, A \subseteq X, J \in \mathcal{J}$ and $B \subseteq Y$, with $D \subseteq(A \times J) \cup(I \times B)$.

It is shown in [10] that $\mathcal{I} \otimes \mathcal{J}$ is an ideal in $X \times Y$, and that if $A$ is open- $\mathcal{I}$ in $(X, \tau, \mathcal{I})$ and $B$ is open- $\mathcal{J}$ in $(Y, \beta, \mathcal{J})$ then $A \times B$ is open- $(\mathcal{I} \otimes \mathcal{J})$.

It is possible to build new $\mathcal{C}$-continuous functions from some previously known ones, as we will show in the two theorems that follow.

Theorem 2.19 If $f:(X, \tau) \rightarrow(Y, \beta, \mathcal{J})$ and $g:(X, \tau) \rightarrow(Z, \gamma, \mathcal{L})$ are $\mathcal{C}$-continuous, then $h:(X, \tau) \rightarrow\left(Y \times Z, \beta \times \gamma,(\mathcal{J} \otimes \mathcal{L})^{\otimes}\right)$, defined by $h(x)=(f(x), g(x))$ for all $x \in X$, is $\mathcal{C}$-continuous.

Proof. If $U \in \beta$ and $V \in \gamma$ then there are $J \in \mathcal{J}$ and $L \in \mathcal{L}$, such that $f^{-1}(U) \subseteq \operatorname{int}\left(f^{-1}(U)\right) \cup f^{-1}(J)$ and $g^{-1}(V) \subseteq \operatorname{int}\left(g^{-1}(V)\right) \cup g^{-1}(L)$.

Now, $h^{-1}(U \times V)=f^{-1}(U) \cap g^{-1}(V) \subseteq\left[\operatorname{int}\left(f^{-1}(U)\right) \cup f^{-1}(J)\right] \cap$ $\left[\operatorname{int}\left(g^{-1}(V)\right) \cup g^{-1}(L)\right]=\operatorname{int}\left[f^{-1}(U) \cap g^{-1}(V)\right] \cup\left[\operatorname{int}\left(f^{-1}(U)\right) \cap g^{-1}(L)\right] \cup$ $\left[f^{-1}(J) \cap \operatorname{int}\left(g^{-1}(V)\right)\right] \cup\left[f^{-1}(J) \cap g^{-1}(L)\right] \subseteq \operatorname{int}\left[h^{-1}(U \times V)\right] \cup h^{-1}(J \times L) \cup$ $h^{-1}(U \times L) \cup h^{-1}(J \times V)=i n t\left[h^{-1}(U \times V)\right] \cup h^{-1}[(J \times L) \cup(U \times L) \cup$ $(J \times V)]$. Moreover $J \times L \in \mathcal{J} \otimes \mathcal{L}$ and $(U \times L) \cup(J \times V) \in \mathcal{J} \otimes$ $\mathcal{L} \subseteq(\mathcal{J} \otimes \mathcal{L})^{\otimes}$. Theorem 2.12 implies that $h$ is $\mathcal{C}$-continuous.

Theorem 2.20 If $f:(X, \tau) \rightarrow(Y, \beta, \mathcal{J})$ is $\mathcal{C}$-continuous and if $\mathcal{J}_{\tau}=$ $\left\{W \subseteq X \times Y\right.$ : there are $U \in \tau$ and $\left.J \in \mathcal{J}_{\text {such that }} W \subseteq U \times J\right\}$, then $\mathcal{J}_{\tau}$ is an ideal in $X \times Y$ and the function $g:(X, \tau) \rightarrow\left(X \times Y, \tau \times \beta,\left(\mathcal{J}_{\tau}\right)^{\otimes}\right)$ , defined by $g(x)=(x, f(x))$, is $\mathcal{C}$-continuous.

Proof. It is easy to be established that $\mathcal{J}_{\tau}$ is an ideal in $X \times Y$. Suppose that $U \in \tau$ and $V \in \beta$. There is a $J \in \mathcal{J}$ such that $f^{-1}(V) \subseteq$ $\operatorname{int}\left(f^{-1}(V)\right) \cup f^{-1}(J)$. Now, $g^{-1}(U \times V)=U \cap f^{-1}(V) \subseteq U \cap\left[i n t\left(f^{-1}(V)\right) \cup f^{-1}(J)\right]=$ $\left[U \cap \operatorname{int}\left(f^{-1}(V)\right)\right] \cup\left[U \cap f^{-1}(J)\right]=\operatorname{int}\left(U \cap f^{-1}(V)\right) \cup g^{-1}(U \times J)=$ 
$\operatorname{int}\left(g^{-1}(U \times V)\right) \cup g^{-1}(U \times J)$, and $U \times J \in \mathcal{J}_{\tau} \subseteq\left(\mathcal{J}_{\tau}\right)^{\otimes}$. Theorem 2.12 implies that $g$ is $\mathcal{C}$-continuous.

Recall that, if $\mathcal{I}$ and $\mathcal{J}$ are ideals in $X$, then the ideal $\mathcal{I} \vee \mathcal{J}$ is the set $\{I \cup J: I \in \mathcal{I}$ and $J \in \mathcal{J}\}$.

Lemma 2.21 If $\mathcal{I}$ and $\mathcal{J}$ are ideals in $X, A \subseteq X$ is open- $\mathcal{I}$ in $(X, \tau, \mathcal{I})$ and $B \subseteq X$ is open- $\mathcal{J}$ in $(X, \tau, \mathcal{J})$, then $A \cap B$ is open- $(\mathcal{I} \vee \mathcal{J})$.

Proof. Since $A \backslash A^{\circ} \in \mathcal{I}$ and $B \backslash B^{\circ} \in \mathcal{J}$, we have that $(A \cap B) \backslash$ int $(A \cap B) \subseteq$ $\left(A \backslash A^{\circ}\right) \cup\left(B \backslash B^{\circ}\right) \in \mathcal{I} \vee \mathcal{J}$, and so $(A \cap B) \backslash \operatorname{int}(A \cap B) \in \mathcal{I} \vee \mathcal{J}$.

If $(X, \tau, \mathcal{I})$ is an ideal space, the set $\tau \cup \mathcal{I}$ is a base for a topology $\tau \oplus \mathcal{I}$ in $X$. It is observed that $\tau \oplus \mathcal{I}=\left\{V \cup I: V \in \tau\right.$ and $\left.I \in \mathcal{I}^{\otimes}\right\}=\tau \oplus \mathcal{I}^{\otimes}[10]$. If $\mathcal{J}$ is an ideal in $Y$ and if $f: X \rightarrow Y$ is a function, we denote the set $\left\{A \subseteq X: A \subseteq f^{-1}(J)\right.$ for some $\left.J \in \mathcal{J}\right\}$ by $\mathcal{I}_{f, \mathcal{J}}$. It is noted that $\mathcal{I}_{f, \mathcal{J}}$ is an ideal in $X$ and that $\left\{f^{-1}(J): J \in \mathcal{J}\right\} \subseteq \mathcal{I}_{f, \mathcal{J}}$.

The following theorem shows some properties of the $\mathcal{C}$-continuous functions, in the case where the codomain is a Hausdorff space.

Theorem 2.22 (1) If $f:(X, \tau) \rightarrow(Y, \beta, \mathcal{J})$ and $g:(X, \tau) \rightarrow(Y, \beta, \mathcal{J})$ are $\mathcal{C}$-continuous functions and if $(Y, \beta)$ is $T_{2}$, the set $A=\{x \in X: f(x)=g(x)\}$ is closed in the space $\left(X, \tau \oplus\left(\mathcal{I}_{f, \mathcal{J}} \vee \mathcal{I}_{g, \mathcal{J}}\right)\right)$.

(2) If $f:(X, \tau) \rightarrow(Y, \beta, \mathcal{J})$ is a $\mathcal{C}$-continuous function and if $(Y, \beta)$ is $T_{2}$, the set $A=\{(u, v) \in X \times X: f(u)=f(v)\}$ is closed in the space $\left(X \times X,(\tau \times \tau) \oplus\left(\mathcal{I}_{f, \mathcal{J}} \otimes \mathcal{I}_{f, \mathcal{J}}\right)\right)$.

Proof. (1) Suppose that $u \in X \backslash A$. There is a $\{U, V\} \subseteq \tau$ such that $f(u) \in U, g(u) \in V$ and $U \cap V=\emptyset$. Then $u \in f^{-1}(U) \cap g^{-1}(V)$, $f^{-1}(U) \cap g^{-1}(V) \cap A=\emptyset$ and, by hypothesis, Lemma 2.21 and Corollary 2.4, $f^{-1}(U) \cap g^{-1}(V)$ is open- $\left(\mathcal{I}_{f, \mathcal{J}} \vee \mathcal{I}_{g, \mathcal{J}}\right)$. Thus $f^{-1}(U) \cap g^{-1}(V) \in$ $\tau \oplus\left(\mathcal{I}_{f, \mathcal{J}} \vee \mathcal{I}_{g, \mathcal{J}}\right)$.

(2) Suppose that $(u, v) \in(X \times X) \backslash A$. There exists $\{U, V\} \subseteq \beta$ such that $f(u) \in U, f(v) \in V$ and $U \cap V=\emptyset$. Thus $(u, v) \in f^{-1}(U) \times f^{-1}(V)$, $A \cap\left[f^{-1}(U) \times f^{-1}(V)\right]=\emptyset$ and $f^{-1}(U) \times f^{-1}(V)$ is open- $\left(\mathcal{I}_{f, \mathcal{J}} \otimes \mathcal{I}_{f, \mathcal{J}}\right)$, and so $f^{-1}(U) \times f^{-1}(V) \in(\tau \times \tau) \oplus\left(\mathcal{I}_{f, \mathcal{J}} \otimes \mathcal{I}_{f, \mathcal{J}}\right)$.

In our last property for this new type of weak continuity, we present the smallest ideal $\mathcal{J}$ in $Y$ for which a given function $f:(X, \tau) \rightarrow(Y, \beta)$ is $\mathcal{C}$-continuous. 
Theorem 2.23 If $f:(X, \tau) \rightarrow(Y, \beta)$ is a function and if $\mathcal{J}_{f}=\left\{B \subseteq Y:\right.$ there is a finite $\Delta_{B} \subseteq \mathcal{P}(X)$ with $\left.B \subseteq \bigcup_{A \in \Delta_{B}}[f(\bar{A}) \backslash \overline{f(A)}]\right\}$ then $\mathcal{J}_{f}$ is the smallest ideal $\mathcal{J}$ in $Y$ such that $f:(X, \tau) \rightarrow(Y, \beta, \mathcal{J})$ is $\mathcal{C}$-continuous.

Proof. It is evident that $\mathcal{J}_{f}$ is an ideal in $Y$. Given that $f(\bar{A}) \backslash \overline{f(A)} \in \mathcal{J}_{f}$, for each $A \subseteq X$, then $f:(X, \tau) \rightarrow\left(Y, \beta, \mathcal{J}_{f}\right)$ is a $\mathcal{C}$-continuous function. Now, suppose that $f:(X, \tau) \rightarrow(Y, \beta, \mathcal{J})$ is $\mathcal{C}$-continuous. If $B \in \mathcal{J}_{f}$ then there is a finite $\Delta_{B} \subseteq \mathcal{P}(X)$ with $B \subseteq \bigcup_{A \in \Delta_{B}}[f(\bar{A}) \backslash \overline{f(A)}]$. Since $f(\bar{A}) \backslash \overline{f(A)} \in \mathcal{J}$, for each $A \in \Delta_{B}$, we can conclude that $B \in \mathcal{J}$. In this way $\mathcal{J}_{f} \subseteq \mathcal{J}$.

The example that follows shows us that $\mathcal{C}$-continuous and $\mathcal{J}$-continuous are independent concepts.

Example 2.24 Let $X=Y=\{a, b\}, \tau=\{\emptyset, X\}, \beta=\{\emptyset,\{a\}, Y\}$ and $f: X \rightarrow Y$ the function defined by $f(a)=b$ and $f(b)=a$. Then:

(1) If $\mathcal{J}=\{\emptyset,\{a\}\}$, it is not a problem to prove that $f$ is $\mathcal{C}$-continuous. However $f(b)=a \in\{a\},\{a\} \in \beta$, but $f(X) \backslash\{a\}=\{b\} \notin \mathcal{J}$. Hence $f$ is not $\mathcal{J}$-continuous.

(2) If $\mathcal{J}=\{\emptyset,\{b\}\}$, it is easy to see that $f$ is $\mathcal{J}$-continuous. Now, since $f(\overline{\{a\}}) \backslash \overline{f(\{a\})}=f(X) \backslash \overline{\{b\}}=\{a\} \notin \mathcal{J}$, we conclude that $f$ is not $\mathcal{C}$ continuous.

Next we show that $\mathcal{C}$-continuous and $\mathcal{J}_{w}$-continuous are independent concepts.

Example 2.25 (1) Suppose that $X=Y=\{a, b, c\}, \beta=\{\emptyset, Y,\{a\},\{b\}$, $\{a, b\}\}, \tau=\{\emptyset, X,\{a, b\},\{b, c\},\{b\}\}, \mathcal{J}=\{\emptyset,\{a\}\}$ and that $f: X \rightarrow Y$ is defined by: $f(a)=a, f(b)=b=f(c)$. It is easy to see that $f$ is $\mathcal{C}$ continuous. Given that $f(a)=a \in\{a\},\{a\} \in \beta, f(\{a, b\}) \backslash \overline{\{a\}}=\{b\} \notin \mathcal{J}$ and $f(X) \backslash \overline{\{a\}}=\{b\}$, then $f$ is not $\mathcal{J}_{w}$-continuous.

(2) Let $X, Y, \tau, \beta$ and $\mathcal{J}$ be as in part (1). If $f: X \rightarrow Y$ is defined by: $f(a)=b=f(c)$, and $f(b)=c$, it is not a problem to prove that $f$ is $\mathcal{J}_{w^{-}}$ continuous. However, given that $f(\overline{\{b\}}) \backslash \overline{f(\{b\})}=\{b, c\} \backslash \overline{\{c\}}=\{b\} \notin \mathcal{J}$, we have that $f$ is not $\mathcal{C}$-continuous. 


\section{3. $\mathcal{C}$-closed and $\mathcal{C}$-open functions}

Through the $\mathcal{I}$-open sets, Abd El Monsef et al. defines the $\mathcal{J}$-open functions. The authors consider the case in which an ideal is defined in the codomain of the function. Under the same requirement, in this section we define the $\mathcal{C}$-open functions, and we show that this new concept is independent of the concept introduced by them. Recall that if $\mathcal{J}$ is an ideal in $Y$, a function $f:(X, \tau) \rightarrow(Y, \beta, \mathcal{J})$ is said to be $\mathcal{J}$-open (resp. $\mathcal{J}$-closed) [1] if, for each $U \in \tau$ (resp. $U$ is closed), we have that $f(U)$ is $\mathcal{J}$-open (resp. $\mathcal{J}$-closed).

Definition 3.1 If $\mathcal{J}$ is an ideal in $Y$, a function $f:(X, \tau) \rightarrow(Y, \beta, \mathcal{J})$ is said to be:

(1) $\mathcal{C}$-closed if, for each $A \subseteq X$, we have that $\overline{f(A)} \backslash f(\bar{A}) \in \mathcal{J}$.

(2) $\mathcal{C}$-open if, for each $A \subseteq X$, we have that $f\left(A^{\circ}\right) \backslash$ int $(f(A)) \in \mathcal{J}$.

It is clear that if $f$ is closed (or open) then $f$ is $\mathcal{C}$-closed (or $\mathcal{C}$-open) and that $f:(X, \tau) \rightarrow(Y, \beta)$ is closed (or open) if and only if $f:(X, \tau) \rightarrow$ $(Y, \beta,\{\emptyset\})$ is $\mathcal{C}$-closed (or $\mathcal{C}$-open). It is immediate to see that $f:(X, \tau) \rightarrow$ $(Y, \beta, \mathcal{J})$ is: (1) $\mathcal{C}$-open if and only if, for each $U \in \tau, f(U)$ is open- $\mathcal{J}$, and $(2) \mathcal{C}$-closed if and only if, for all closed set $F \subseteq X, f(F)$ is closed$\mathcal{J}$. Moreover, if $f$ is $\mathcal{C}$-open then $f:(X, \tau) \rightarrow(Y, \beta \oplus \mathcal{J})$ is open, and $f:(X, \tau) \rightarrow\left(Y, \beta, \mathcal{J}^{\otimes}\right)$ is $\mathcal{C}$-open if and only if $f:(X, \tau) \rightarrow(Y, \beta \oplus \mathcal{J})$ is open.

\section{Examples 3.2}

(1) Each function $f:(X, \tau) \rightarrow(Y, \beta, \mathcal{J}=\mathcal{P}(Y))$ is $\mathcal{C}$-closed and $\mathcal{C}$-open.

(2) If $\beta=\{\emptyset, \mathbf{R},\{0\}\}$ and $\mathcal{J}=\mathcal{P}(\{-1,0,1\})$ then the function $f$ :

$(\mathbf{R}, \mathcal{U}) \rightarrow(\mathbf{R}, \beta, \mathcal{J})$ defined by

$$
f(x)=\left\{\begin{array}{c}
1, \text { if } x>0 \\
0, \text { if } x=0 \\
-1, \text { if } x<0
\end{array}\right.
$$

is $\mathcal{C}$-open, but $f$ is not open. Moreover $f$ is not $\mathcal{C}$-closed, because $\overline{f(\mathbf{R})} \backslash f(\overline{\mathbf{R}})$ $=\mathbf{R} \backslash\{-1,0,1\} \notin \mathcal{J}$.

(3) If $\beta=\{\emptyset, \mathbf{R}\} \cup\{(r, \infty): r \in \mathbf{R}\}$ and $\mathcal{J}=\mathcal{P}((-\infty, 1])$, then the function $f:(\mathbf{R}, \mathcal{U}) \rightarrow(\mathbf{R}, \beta, \mathcal{J})$, defined as in example $(2)$, is not closed, but $f$ is $\mathcal{C}$-closed since if $F \subseteq \mathbf{R}$ then $\overline{f(F)} \subseteq(-\infty, 1]$, and so $\overline{f(F)} \backslash f(\bar{F}) \in \mathcal{J}$.

(4) Consider the function $f:(\mathbf{R}, \mathcal{U}) \rightarrow(\mathbf{R}, \mathcal{U}, \mathcal{J}=\mathcal{P}(\{0\}))$ defined by $f(x)=[x]$, where $[x]$ denotes the integer part of $x$. 
Since $f(\operatorname{int}([1,2])) \backslash \operatorname{int}(f([1,2]))=\{1\} \notin \mathcal{J}$, we conclude that $f$ is not $\mathcal{C}$-open.

Since that open $\rightarrow \mathcal{C}$-open, and that $\mathcal{J}$-open and open are independent concepts [1], we have that $\mathcal{C}$-open $\not \rightarrow \mathcal{J}$-open. The next example shows that $\mathcal{J}$-open $\nrightarrow \mathcal{C}$-open.

Example 3.3 Suppose that $X=Y=\{a, b, c, d\}$ and that $f: X \rightarrow Y$ is defined by $f(x)=c$, for each $x \in X$. Let $\tau=\{\emptyset, X,\{a\},\{b\},\{a, b\}\}$, $\beta=\{\emptyset, Y,\{a, d\},\{b, c\}\}$ and $\mathcal{J}=\{\emptyset,\{b\},\{d\},\{b, d\}\}$. Given that $\{a\} \in \tau$ and $f(\{a\}) \backslash \operatorname{int}(f(\{a\}))=\{c\} \notin \mathcal{J}$, we have that $f$ is not $\mathcal{C}$-open. Since, for each $U \in \tau \backslash\{\emptyset\}, f(U)=\{c\} \subseteq\{b, c\}=\operatorname{int}\left[(f(U))^{*}\right]$, we conclude that $f$ is $\mathcal{J}$-open.

Theorem 3.4 Suppose that $(X, \tau)$ and $(Y, \beta)$ are topological spaces, $\mathcal{B}$ is a base for $\tau$ and that $\mathcal{J}$ is an ideal in $Y$. Then the function $f:(X, \tau) \rightarrow\left(Y, \beta, \mathcal{J}^{\otimes}\right)$ is $\mathcal{C}$-open if and only $f(B)$ is open- $\mathcal{J}^{\otimes}$, for each $B \in \mathcal{B}$.

Proof. $(\rightarrow)$ It is clear.

$(\leftarrow)$ If $V \in \tau$, there exists $\left\{V_{\alpha}: \alpha \in \Lambda\right\} \subseteq \mathcal{B}$ such that $V=\bigcup_{\alpha \in \Lambda} V_{\alpha}$. For each $\alpha \in \Lambda$ there exists $J_{\alpha} \in \mathcal{J}^{\otimes}$ such that $f\left(V_{\alpha}\right)=\operatorname{int}\left(f\left(V_{\alpha}\right)\right) \cup J_{\alpha}$. Then $f(V)=\bigcup_{\alpha \in \Lambda} f\left(V_{\alpha}\right)=\bigcup_{\alpha \in \Lambda} \operatorname{int}\left(f\left(V_{\alpha}\right)\right) \cup \bigcup_{\alpha \in \Lambda} J_{\alpha} \subseteq \operatorname{int}(f(V)) \cup \bigcup_{\alpha \in \Lambda} J_{\alpha}$. Hence $f(V) \backslash \operatorname{int}(f(V)) \in \mathcal{J}^{\otimes}$.

Theorem $3.5(1)$ Suppose that $f:(X, \tau) \rightarrow(Y, \beta, \mathcal{J})$ and $g:(Y, \beta) \rightarrow$ $(Z, \gamma, \mathcal{L})$ are $\mathcal{C}$-open and that $g(\mathcal{J}) \subseteq \mathcal{L}$. Then $g \circ f$ is $\mathcal{C}$-open.

(2) If $f:(X, \tau) \rightarrow(Y, \beta)$ is open and if $g:(Y, \beta) \rightarrow(Z, \gamma, \mathcal{L})$ is $\mathcal{C}$-open, then $g \circ f$ is $\mathcal{C}$-open.

Proof. (1) If $U \in \tau$ then there is a $J \in \mathcal{J}$ such that $f(U) \backslash$ int $(f(U))=J$. Then $f(U)=\operatorname{int}(f(U)) \cup J$. There exists $L \in \mathcal{L}$ with $g(\operatorname{int}(f(U)))=$ int $(g($ int $(f(U)))) \cup L$.

Hence $g(f(U))=g(\operatorname{int}(f(U))) \cup g(J)=\operatorname{int}(g(\operatorname{int}(f(U)))) \cup L \cup$ $g(J) \subseteq \operatorname{int}(g(f(U))) \cup L \cup g(J)$, and $L \cup g(J) \in \mathcal{L}$.

This implies that $(g \circ f)(U) \backslash$ int $((g \circ f)(U)) \in \mathcal{L}$.

(2) It is a consequence of (1).

The following example shows that the composition of $\mathcal{C}$-open functions may not be a $\mathcal{C}$-open function. 
Example 3.6 Suppose that $X=Y=Z=\{a, b, c, d\}$ and that $f: X \rightarrow Y$ and $g: Y \rightarrow Z$ are functions defined by $f(a)=b=f(b), f(c)=c=f(d)$ and $g(a)=a, g(b)=c, g(c)=b, g(d)=d$.

If $\beta=\{\emptyset, Y,\{b\},\{a, d\},\{a, b, d\}\}, \tau=\{\emptyset, X,\{a, b, c\},\{a, c, d\},\{a, c\}\}$, $\gamma=\{\emptyset, Z,\{c\}\}, \mathcal{J}=\{\emptyset,\{c\}\}, \mathcal{L}=\{\emptyset,\{a\},\{d\},\{a, d\}\}$, we have that $g \circ f$ is not $\mathcal{C}$-open, because $(g \circ f)(\{a, b, c\}) \backslash \operatorname{int}[(g \circ f)(\{a, b, c\})]=\{b\} \notin \mathcal{L}$. However $f$ and $g$ are $\mathcal{C}$-open.

Theorem 3.7 If $\mathcal{I}$ is an ideal in $X$ then the function $f:(X, \tau) \rightarrow$ $(Y, \beta, f(\mathcal{I}))$ is $\mathcal{C}$-closed if and only if, for each closed-I set $A \subseteq X$, it is true that $f(A)$ is closed- $f(\mathcal{I})$.

Proof. $(\rightarrow)$ Suppose that $A \subseteq X$ is closed-I. Since $\bar{A} \backslash A \in \mathcal{I}$ and $f(\bar{A}) \backslash f(A) \subseteq f(\bar{A} \backslash A) \in f(\mathcal{I})$, then $f(\bar{A}) \backslash f(A) \in f(\mathcal{I})$. Now, given that $f$ is $\mathcal{C}$-closed, we have that $\overline{f(\bar{A})} \backslash f(\bar{A}) \in f(\mathcal{I})$. Moreover, $\overline{f(A)} \backslash f(A) \subseteq$ $\overline{f(\bar{A})} \backslash f(A) \subseteq[\overline{f(\bar{A})} \backslash f(\bar{A})] \cup[f(\bar{A}) \backslash f(A)] \in f(\mathcal{I})$, and so $\overline{f(A)} \backslash f(A) \in$ $f(\mathcal{I})$.

$(\leftarrow)$ It is clear.

\section{Some applications of $\mathcal{C}$-continuous, $\mathcal{C}$-closed and $\mathcal{C}$-open functions}

In this section we present some applications of these three new type of functions, mainly related to compactness and separability, which constitute generalizations of well-known results of the general topology. If $(X, \tau, \mathcal{I})$ is an ideal space, the set $\overline{\mathcal{I}}=\{A \subseteq X: A \subseteq \bar{I}$, for some $I \in \mathcal{I}\}$ is an ideal in $X$. Moreover, it is clear that if $J \in \overline{\mathcal{I}}$ then $\bar{J} \in \overline{\mathcal{I}}$.

Recall that a topological space $(X, \tau)$ is said to be quasi-H-closed, or simply QHC [11], if for each open cover $\left\{V_{\alpha}\right\}_{\alpha \in \Lambda}$ of $X$, there exists a finite $\Lambda_{0} \subseteq \Lambda$ with $X=\bigcup_{\alpha \in \Lambda_{0}} \overline{V_{\alpha}}$. An ideal space $(X, \tau, \mathcal{I})$ is defined to be: (1) $\mathcal{I}$ compact [7] if for all open cover $\left\{V_{\alpha}\right\}_{\alpha \in \Lambda}$ of $X$, there exists a finite $\Lambda_{0} \subseteq \Lambda$ such that $X \backslash \bigcup_{\alpha \in \Lambda_{0}} V_{\alpha} \in \mathcal{I}$, (2) $\mathcal{I}$-QHC [3] if for all open cover $\left\{V_{\alpha}\right\}_{\alpha \in \Lambda}$ of $X$, there exists a finite $\Lambda_{0} \subseteq \Lambda$ such that $X \backslash \bigcup_{\alpha \in \Lambda_{0}} \overline{V_{\alpha}} \in \mathcal{I}$, and (3) $\mathcal{I}$-normal 
[12] if, for each pair of disjoint closed sets $F$ and $G$, there are disjoint open sets $U$ and $V$ such that $F \backslash U \in \mathcal{I}$ and $G \backslash V \in \mathcal{I}$.

Theorem 4.1 If $f:(X, \tau) \rightarrow(Y, \beta, \mathcal{J})$ is sobreyective and $\mathcal{C}$-continuous and if $\left(X, \tau \oplus \mathcal{I}_{f, \mathcal{J}}\right)$ is compact, then $(Y, \beta, \mathcal{J})$ is $\mathcal{J}$-compact.

Proof. If $\left\{V_{\alpha}\right\}_{\alpha \in \Lambda}$ is an open cover of $Y$ then $X=\bigcup_{\alpha \in \Lambda} f^{-1}\left(V_{\alpha}\right)$. For each $\alpha \in \Lambda$, there exists $J_{\alpha} \in \mathcal{J}$ such that $f^{-1}\left(V_{\alpha}\right) \subseteq \operatorname{int}\left[f^{-1}\left(V_{\alpha}\right)\right] \cup f^{-1}\left(J_{\alpha}\right)$. Thus $X=\bigcup_{\alpha \in \Lambda}\left\{\right.$ int $\left.\left[f^{-1}\left(V_{\alpha}\right)\right] \cup f^{-1}\left(J_{\alpha}\right)\right\}$, with $\operatorname{int}\left[f^{-1}\left(V_{\alpha}\right)\right] \cup f^{-1}\left(J_{\alpha}\right) \in$ $\tau \oplus \mathcal{I}_{f, \mathcal{J}}$, for each $\alpha \in \Lambda$. There exists a finite $\Lambda_{0} \subseteq \Lambda$ such that $X=$ $\bigcup_{\alpha \in \Lambda_{0}}\left\{\right.$ int $\left.\left[f^{-1}\left(V_{\alpha}\right)\right] \cup f^{-1}\left(J_{\alpha}\right)\right\}$. This implies that $X=\left(\bigcup_{\alpha \in \Lambda_{0}} f^{-1}\left(V_{\alpha}\right)\right) \cup$ $\bigcup_{\alpha \in \Lambda_{0}} f^{-1}\left(J_{\alpha}\right)$. Hence $Y=\left(\bigcup_{\alpha \in \Lambda_{0}} V_{\alpha}\right) \cup \bigcup_{\alpha \in \Lambda_{0}} J_{\alpha}$, and so $Y \backslash \bigcup_{\alpha \in \Lambda_{0}} V_{\alpha} \in \mathcal{J}$.

Theorem 4.2 If $f:(X, \tau) \rightarrow(Y, \beta, \mathcal{J})$ is sobreyective and $\mathcal{C}$-continuous and if $\left(X, \tau \oplus \mathcal{I}_{f, \mathcal{J}}\right)$ is $\mathrm{QHC}$, then $(Y, \beta, \overline{\mathcal{J}})$ is $\overline{\mathcal{J}}-\mathrm{QHC}$.

Proof. Let $\lambda=\tau \oplus \mathcal{I}_{f, \mathcal{J}}$ be. Suppose that $\left\{V_{\alpha}\right\}_{\alpha \in \Lambda}$ is an open cover of $Y$. For each $\alpha \in \Lambda$ there exists $J_{\alpha} \in \mathcal{J}$ such that $f^{-1}\left(V_{\alpha}\right) \subseteq f^{-1}\left(J_{\alpha}\right) \cup$ int $_{\tau}\left(f^{-1}\left(V_{\alpha}\right)\right)$. Hence $X=\bigcup_{\alpha \in \Lambda}\left[f^{-1}\left(J_{\alpha}\right) \cup i n t_{\tau}\left(f^{-1}\left(V_{\alpha}\right)\right)\right]$. Given that $(X, \lambda)$ is QHC, we have that $X=\bigcup_{\alpha \in \Lambda_{0}}\left[a d h_{\lambda}\left(f^{-1}\left(J_{\alpha}\right)\right) \cup a d h_{\lambda}\left(i n t_{\tau}\left(f^{-1}\left(V_{\alpha}\right)\right)\right)\right]$, for some finite $\Lambda_{0} \subseteq \Lambda$. But $a d h_{\lambda}(A) \subseteq a d h_{\tau}(A)$, for each $A \subseteq X$. This allows us to conclude that $X=\bigcup_{\alpha \in \Lambda_{0}}\left[a d h_{\tau}\left(f^{-1}\left(J_{\alpha}\right)\right) \cup a d h_{\tau}\left(f^{-1}\left(V_{\alpha}\right)\right)\right]$. Thus $Y=\bigcup_{\alpha \in \Lambda_{0}}\left[f\left(a d h_{\tau}\left(f^{-1}\left(J_{\alpha}\right)\right)\right) \cup f\left(a d h_{\tau}\left(f^{-1}\left(V_{\alpha}\right)\right)\right)\right]$. For each $\alpha \in$ $\Lambda_{0}$ there exist $\left\{L_{\alpha}, M_{\alpha}\right\} \subseteq \mathcal{J}$ such that $f\left(a d h_{\tau}\left(f^{-1}\left(V_{\alpha}\right)\right)\right) \subseteq a d h_{\beta}\left[f\left(f^{-1}\left(V_{\alpha}\right)\right)\right] \cup$ $L_{\alpha}=a d h_{\beta}\left(V_{\alpha}\right) \cup L_{\alpha}$ and $f\left(a d h_{\tau}\left(f^{-1}\left(J_{\alpha}\right)\right)\right) \subseteq a d h_{\beta}\left[f\left(f^{-1}\left(J_{\alpha}\right)\right)\right] \cup M_{\alpha}=$ $a d h_{\beta}\left(J_{\alpha}\right) \cup M_{\alpha}$. In consequence, $Y=\bigcup_{\alpha \in \Lambda_{0}} a d h_{\beta}\left(V_{\alpha}\right) \cup \bigcup_{\alpha \in \Lambda_{0}}\left[a d h_{\beta}\left(J_{\alpha}\right) \cup L_{\alpha} \cup M_{\alpha}\right]$ and so $Y \backslash \bigcup_{\alpha \in \Lambda_{0}} a d h_{\beta}\left(V_{\alpha}\right) \in \overline{\mathcal{J}}$.

Theorem 4.3 If $f:(X, \tau) \rightarrow(Y, \beta, \mathcal{J})$ is $\mathcal{C}$-continuous then:

(1) If $\left\{x_{n}\right\}_{n=1}^{\infty}$ is a succession in $X, a \in X$ and $x_{n} \rightarrow a$, then either $\{f(a)\} \in \mathcal{J}$ or $f\left(x_{n}\right) \rightarrow f(a)$.

(2) If $\mathcal{F}$ is a filter in $X, a \in X$ and $\mathcal{F} \rightarrow a$ then either $\{f(a)\} \in \mathcal{J}$ or $f(\mathcal{F}) \rightarrow f(a)$. 


\section{Proof.}

(1) Suppose that $\{f(a)\} \notin \mathcal{J}$. If $W \in \beta$ and $f(a) \in W$, there exists $J \in \mathcal{J}$ such that $a \in f^{-1}(W) \subseteq f^{-1}(J) \cup \operatorname{int}\left(f^{-1}(W)\right)$. Since $\{f(a)\} \notin \mathcal{J}$ then $a \in \operatorname{int}\left(f^{-1}(W)\right)$, and so there is a $N \in \mathbf{Z}^{+}$such that, if $n \geq N$ then $x_{n} \in \operatorname{int}\left(f^{-1}(W)\right)$. Thus, if $n \geq N$ we have that $f\left(x_{n}\right) \in W$.

(2) Suppose that $\{f(a)\} \notin \mathcal{J}$. If $W \in \beta$ and $f(a) \in W$, there exists $J \in \mathcal{J}$ such that $a \in f^{-1}(W) \subseteq f^{-1}(J) \cup \operatorname{int}\left(f^{-1}(W)\right)$. Since $\{f(a)\} \notin \mathcal{J}$ then $a \in \operatorname{int}\left(f^{-1}(W)\right)$, and so int $\left(f^{-1}(W)\right) \in \mathcal{F}$. This implies that $f\left(\right.$ int $\left.\left(f^{-1}(W)\right)\right) \in f(\mathcal{F})$. Given that $f\left(\right.$ int $\left.\left(f^{-1}(W)\right)\right) \subseteq W$, we have that $W \in f(\mathcal{F})$.

Theorem 4.4 If $f:(X, \tau) \rightarrow(Y, \beta, \mathcal{J})$ is continuous, sobreyective and $\mathcal{C}$ -closed, and if $(X, \tau)$ is normal, then $(Y, \beta, \mathcal{J})$ is $\mathcal{J}$-normal.

Proof. Suppose that $F$ and $G$ are disjoint closed subsets of $Y$. There exist disjoint $U \in \tau$ and $V \in \tau$, with $f^{-1}(F) \subseteq U$ and $f^{-1}(G) \subseteq$ $V$. Then $f(X \backslash U) \subseteq Y \backslash F$ and $f(X \backslash V) \subseteq Y \backslash G$. Now, $[Y \backslash \overline{f(X \backslash U)}] \cap$ $[Y \backslash \overline{f(X \backslash V)}]=Y \backslash \overline{f[X \backslash(U \cap V)]}=\emptyset$. Moreover

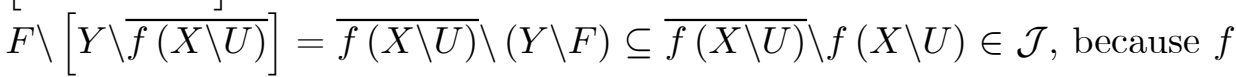
is $\mathcal{C}$-closed. Hence $F \backslash[Y \backslash \overline{f(X \backslash U)}] \in \mathcal{J}$. Similarly $G \backslash[Y \backslash \overline{f(X \backslash V)}] \in \mathcal{J}$.

Definition 4.5 The ideal space $(X, \tau, \mathcal{I})$ is said to be separable- $\mathcal{I}$ if there exists a countable $D \subseteq X$ such that $X \backslash \bar{D} \in \mathcal{I}$.

It is noted that separable $\rightarrow$ separable- $\mathcal{I}$ and that $(X, \tau)$ is separable if and only if $(X, \tau,\{\emptyset\})$ is separable- $\{\emptyset\}$.

\section{Example 4.6}

(1) The space $(\mathbf{R}, \mathcal{P}(\mathbf{R}), \mathcal{I}=\mathcal{P}(\mathbf{R}))$ is separable- $\mathcal{I}$, but $(\mathbf{R}, \mathcal{P}(\mathbf{R}))$ is not separable. (2) Let $\beta$ be the topology in $\mathbf{R}$ given by: $V \in \beta$ if and only if, for each $r \in \mathbf{R}$, if $r \in V \cap \mathbf{Q}$ then there exists $\epsilon>0$ such that $(r-\epsilon, r+\epsilon) \subseteq V$. We have that the space $(\mathbf{R}, \beta, \mathcal{I}=\mathcal{P}(\mathbf{R} \backslash \mathbf{Q}))$ is separable- $\mathcal{I}$ since $\mathbf{R} \backslash \overline{\mathbf{Q}}=\mathbf{R} \backslash \mathbf{Q} \in \mathcal{I}$. Besides $(\mathbf{R}, \beta)$ is not separable. (3) If $\zeta=\{\emptyset\} \cup\{A \subseteq \mathbf{R}: \mathbf{R} \backslash A$ is countable $\}$ then $\left(\mathbf{R}, \zeta, \mathcal{I}=\mathcal{I}_{c}(\mathbf{R})\right)$ is not separable- $\mathcal{I}$, because if $A \subseteq \mathbf{R}$ is countable we have that $\bar{A}=A$ and so $\mathbf{R} \backslash \bar{A} \notin \mathcal{I}$.

\section{Theorem 4.7}

(1) If $f:(X, \tau) \rightarrow(Y, \beta, \mathcal{J})$ is sobreyective and $\mathcal{C}$-continuous, and if $(X, \tau)$ is separable then $(Y, \beta, \mathcal{J})$ is separable- $\mathcal{J}$. 
(2) If $\mathcal{I}$ is an ideal in $X, f:(X, \tau) \rightarrow(Y, \beta, f(\mathcal{I}))$ is sobreyective and $\mathcal{C}$ -continuous, and if $(X, \tau, \mathcal{I})$ is separable- $\mathcal{I}$, then $(Y, \beta, f(\mathcal{I}))$ is separable$f(\mathcal{I})$.

(3) If $(X, \tau, \mathcal{I})$ is separable- $\mathcal{I}$ and $V \in \tau$, then $\left(V, \tau_{V}, \mathcal{I}_{V}\right)$ is separable$\mathcal{I}_{V}$.

(4) If $(X, \tau, \mathcal{I})$ is separable- $\mathcal{I}$ and $(Y, \beta, \mathcal{J})$ is separable- $\mathcal{J}$ then the space $(X \times Y, \tau \times \beta, \mathcal{I} \otimes \mathcal{J})$ is separable- $(\mathcal{I} \otimes \mathcal{J})$.

\section{Proof.}

(1) There exists $D \subseteq X$, countable, with $\bar{D}=X$. Hence $Y \backslash \overline{f(D)}=$ $f(\bar{D}) \backslash \overline{f(D)} \in \mathcal{J}$, because $f$ is $\mathcal{C}$-continuous. Moreover $f(D)$ is countable.

(2) There is a countable $D \subseteq X$ with $X \backslash \bar{D} \in \mathcal{I}$. Given that $Y \backslash f(\bar{D}) \subseteq f(X \backslash \bar{D}) \in f(\mathcal{I})$ and $f(\bar{D}) \backslash \overline{f(D)} \in f(\mathcal{I})$, then $Y \backslash \overline{f(D)} \in$ $f(\mathcal{I})$.

(3) There exists a countable $D \subseteq X$ such that $X \backslash \bar{D} \in \mathcal{I}$.

Since $V \backslash a d h_{\tau_{V}}(V \cap D)=V \backslash[\overline{V \cap D} \cap V]=V \cap(X \backslash \bar{D})$ and $X \backslash \bar{D} \in$ $\mathcal{I}$, we have that $V \backslash a d h_{\tau_{V}}(V \cap D) \in \mathcal{I}_{V}$.

(4) There are countable sets $D \subseteq X$ and $E \subseteq Y$ such that $X \backslash \bar{D} \in \mathcal{I}$ and $Y \backslash \bar{E} \in \mathcal{J}$.

Given that $(X \times Y) \backslash \overline{D \times E}=(X \times Y) \backslash(\bar{D} \times \bar{E})=[(X \backslash \bar{D}) \times Y] \cup$ $[X \times(Y \backslash \bar{E})]$, we have that $(X \times Y) \backslash \overline{D \times E} \in \mathcal{I} \otimes \mathcal{J}$.

Definition 4.8 The space $(X, \tau, \mathcal{I})$ is said to be regular- $\mathcal{I}$ if for each $U \in \tau$ and $x \in U$, it is true that either $\{x\} \in \mathcal{I}$ or there is a $V \in \tau$ such that $x \in V$ and $\bar{V} \subseteq U$.

It is clear that regular $\rightarrow$ regular- $\mathcal{I}$ and that $(X, \tau)$ is regular if and only if $(X, \tau,\{\emptyset\})$ is regular- $\{\emptyset\}$.

\section{Example 4.9}

(1) Let $\lambda$ be the topology in $\mathbf{R}$ in which the nboods of any nonzero point being as in the usual topology, while nboods of 0 will have the form $U \backslash\{1,1 / 2,1 / 3, \ldots\}$ where $U$ is a nbood of 0 in that usual topology. It is known that $\{1,1 / 2,1 / 3, \ldots\}$ is a closed set and that $(\mathbf{R}, \lambda)$ is not regular. However $(\mathbf{R}, \lambda, \mathcal{I}=\mathcal{P}(\{0\}))$ is regular- $\mathcal{I}$. Indeed, suppose that $U \in \lambda$ and $a \in U$.

(i) If $a=0$ then $\{a\} \in \mathcal{I}$.

(ii) If $a \neq 0$, there is $r \in(0,|a|)$ such that $(a-r, a+r) \subseteq U$. It is very easy to see that $a d h_{\lambda}((a-r / 2, a+r / 2))=[a-r / 2, a+r / 2] \subseteq U$. 
(2) The space $(\mathbf{R}, \lambda, \mathcal{I}=\mathcal{P}(\{1\}))$ is not regular- $\mathcal{I}$, because we have that $0 \in \mathbf{R} \backslash\{1,1 / 2,1 / 3, \ldots\},\{0\} \notin \mathcal{I}$ and there is no $V \in \lambda$ with $0 \in V \subseteq \bar{V} \subseteq$ $\mathbf{R} \backslash\{1,1 / 2,1 / 3, \ldots\}$.

(3) If $\mathcal{I}$ is an admissible ideal in $X$, then each space $(X, \tau, \mathcal{I})$ is regular- $\mathcal{I}$.

\section{Theorem 4.10}

(1) If $f:(X, \tau) \rightarrow(Y, \beta, \mathcal{J})$ is sobreyective, open, closed and $\mathcal{C}$ continuous, and if $(X, \tau)$ is regular then $(Y, \beta, \mathcal{J})$ is regular- $\mathcal{J}$.

(2) If $\mathcal{I}$ is an ideal in $X, f:(X, \tau) \rightarrow(Y, \beta, f(\mathcal{I}))$ is sobreyective, open, closed and $\mathcal{C}$-continuous, and if $(X, \tau, \mathcal{I})$ is regular- $\mathcal{I}$ then $(Y, \beta, f(\mathcal{I}))$ is regular- $f(\mathcal{I})$.

(3) If $(X, \tau, \mathcal{I})$ is regular- $\mathcal{I}$ and $(Y, \beta, \mathcal{J})$ is regular- $\mathcal{J}$ then the space $(X \times Y, \tau \times \beta, \mathcal{I} \otimes \mathcal{J})$ is regular- $(\mathcal{I} \otimes \mathcal{J})$.

\section{Proof.}

(1) If $W \in \beta$ and $y_{0} \in W$, there is a $x_{0} \in X$ such that $f\left(x_{0}\right)=y_{0}$. There exists $J \in \mathcal{J}$ with $x_{0} \in f^{-1}(W) \subseteq \operatorname{int}\left(f^{-1}(W)\right) \cup f^{-1}(J)$. If $x_{0} \in f^{-1}(J)$ then $\left\{y_{0}\right\} \in \mathcal{J}$. If $x_{0} \in \operatorname{int}\left(f^{-1}(W)\right)$, there is a $U \in \tau$ such that $x_{0} \in U$ and $\bar{U} \subseteq \operatorname{int}\left(f^{-1}(W)\right)$. In this case $y_{0} \in f(U)$ and $\overline{f(U)} \subseteq f(\bar{U}) \subseteq f\left(\right.$ int $\left.\left(f^{-1}(W)\right)\right) \subseteq f\left(\left(f^{-1}(W)\right)\right)=W$, with $f(U) \in \beta$. (2) Suppose that $W \in \beta$ and $f\left(x_{0}\right) \in W$. There exists $I \in \mathcal{I}$ such that $x_{0} \in f^{-1}(W) \subseteq \operatorname{int}\left(f^{-1}(W)\right) \cup f^{-1}(f(I))$, since $f$ is $\mathcal{C}$-continuous. If $x_{0} \in f^{-1}(f(I))$ then $\left\{f\left(x_{0}\right)\right\} \in f(\mathcal{I})$. If $x_{0} \in \operatorname{int}\left(f^{-1}(W)\right)$ then either $\left\{x_{0}\right\} \in \mathcal{I}$, and so $\left\{f\left(x_{0}\right)\right\} \in f(\mathcal{I})$, or there exists $U \in \tau$ such that $x_{0} \in U \subseteq \bar{U} \subseteq \operatorname{int}\left(f^{-1}(W)\right)$, and so $f\left(x_{0}\right) \in f(U) \subseteq \overline{f(U)} \subseteq f(\bar{U}) \subseteq$ $f\left(\operatorname{int}\left(f^{-1}(W)\right)\right) \subseteq W$, with $f(U) \in \beta$.

(3) Suppose that $W \in \tau \times \beta$ and $(x, y) \in W$. There are $U \in \tau$ and $V \in \beta$ such that $(x, y) \in U \times V \subseteq W$. We have that either $\{x\} \in \mathcal{I}$ or there is a $U_{1} \in \tau$ with $x \in U_{1}$ and $\overline{U_{1}} \subseteq U$. Moreover, either $\{y\} \in \mathcal{J}$ or there is a $V_{1} \in \beta$ with $y \in V_{1}$ and $\overline{V_{1}} \subseteq V$.

(a) If $\{x\} \in \mathcal{I}$ or $\{y\} \in \mathcal{J}$, it is clear that $\{(x, y)\}=\{x\} \times\{y\} \in \mathcal{I} \otimes \mathcal{J}$.

(b) If there are $U_{1} \in \tau$ and $V_{1} \in \beta$, such that $x \in U_{1}, \overline{U_{1}} \subseteq U, y \in V_{1}$ and $\overline{V_{1}} \subseteq V$, then $(x, y) \in U_{1} \times V_{1}$ and $\overline{U_{1} \times V_{1}}=\overline{U_{1}} \times \overline{V_{1}} \subseteq U \times V \subseteq W$.

Theorem 4.11 If $(X, \tau)$ is $T_{2}$ and if $f:(X, \tau) \rightarrow(Y, \beta, \mathcal{J})$ is biyective and $\mathcal{C}$-open, then $(Y, \beta \oplus \mathcal{J})$ is $T_{2}$.

Proof. Suppose that $\{u, v\} \subseteq Y$ and $u \neq v$. There exists $\{a, b\} \subseteq X$ with $f(a)=u$ and $f(b)=v$. Now, there is a $\{U, V\} \subseteq \tau$ such that $a \in U, b \in V$ 
and $U \cap V=\emptyset$. Thus $u \in f(U), v \in f(V), \quad f(U) \cap f(V)=\emptyset$, with $f(U)$ and $f(V)$ open- $\mathcal{J}$. So that $\{f(U), f(V)\} \subseteq \beta \oplus \mathcal{J}$.

\section{D-Continuous functions}

Other forms of continuity via ideals were defined and studied by Abd El Monsef et al. and by Kaniewski et al. In both cases the considered ideal is defined in the domain of the function. Under the same hypothesis, in this section we define the $\mathcal{D}$-continuous functions, a new and natural generalization of the concept of continuity, which turns out to be independent of the concepts introduced by these authors.

Recall that if $\mathcal{I}$ is an ideal in $X$ then a function $f:(X, \tau, \mathcal{I}) \rightarrow(Y, \beta)$ is said to be $\mathcal{I}$-continuous [1] [El-Monsef form] if, for each $V \in \beta, f^{-1}(V)$ is $\mathcal{I}$-open, that is, $f^{-1}(V) \subseteq$ int $\left[\left(f^{-1}(V)\right)^{*}\right]$. On the other hand, $f$ is defined to be $\mathcal{I}$-continuous [6] [Kaniewski form] if, for each $V \in \beta$, there are $U \in \tau$ and $I \in \mathcal{I}$, such that $f^{-1}(V)=U \backslash I$.

Definition 5.1 If $\mathcal{I}$ is an ideal in $X$, a function $f:(X, \tau, \mathcal{I}) \rightarrow(Y, \beta)$ is said to be $\mathcal{D}$-continuous if, for each $V \in \beta$, we have that $f^{-1}(V)$ is open- $\mathcal{I}$, that is, $f^{-1}(V) \backslash$ int $\left(f^{-1}(V)\right) \in \mathcal{I}$.

It is observed that if $f$ is continuous then $f$ is $\mathcal{D}$-continuous, and that $f:(X, \tau) \rightarrow(Y, \beta)$ is continuous if and only if $f:(X, \tau,\{\emptyset\}) \rightarrow(Y, \beta)$ is $\mathcal{D}$-continuous.

The following theorem establishes a relationship between $\mathcal{C}$-continuity and $\mathcal{D}$-continuity.

\section{Theorem 5.2}

(1) The function $f:(X, \tau, \mathcal{I}) \rightarrow(Y, \beta)$ is $\mathcal{D}$-continuous if and only $f^{-1}(F)$ is closed- $\mathcal{I}$, for each closed set $F \subseteq Y$.

(2) If $f:(X, \tau, \mathcal{I}) \rightarrow(Y, \beta)$ is a $\mathcal{D}$-continuous function, then the function $f:(X, \tau) \rightarrow(Y, \beta, f(\mathcal{I}))$ is $\mathcal{C}$-continuous.

\section{Proof.}

(1) It is clear.

(2) If $V \in \beta$ there is a $I \in \mathcal{I}$ such that $f^{-1}(V)=\operatorname{int}\left(f^{-1}(V)\right) \cup I$ $\subseteq$ int $\left(f^{-1}(V)\right) \cup f^{-1}(f(I))$, with $f(I) \in f(\mathcal{I})$.

Corollary 2.4 implies that $f:(X, \tau) \rightarrow(Y, \beta, f(\mathcal{I}))$ is $\mathcal{C}$-continuous. 


\section{Example 5.3}

(1) If $\mathcal{U}$ is the usual topology in $\mathbf{R}$, the identity function $f:(\mathbf{R}, \mathcal{U}, \mathcal{I}=\mathcal{P}(\mathbf{R})) \rightarrow$ $(\mathbf{R}, \mathcal{P}(\mathbf{R}))$ is $\mathcal{D}$-continuous, but $f$ is not continuous.

(2) The function $f:(\mathbf{R}, \mathcal{U}, \mathcal{I}=\mathcal{P}(\mathbf{Z})) \rightarrow(\mathbf{R}, \mathcal{U})$ given by $f(x)=[x]$, where $[x]$ is the integer part of $x$, is $\mathcal{D}$-continuous, because if $B \subseteq \mathbf{R}$ then $f^{-1}(B)=\bigcup_{n \in B \cap \mathbf{Z}}[n, n+1)$, and given that $\{[n, n+1): n \in B \cap \mathbf{Z}\}$ is locally finite in $(\mathbf{R}, \mathcal{U})$, we have that $\overline{\bigcup_{n \in B \cap \mathbf{Z}}[n, n+1)}=\bigcup_{n \in B \cap \mathbf{Z}} \overline{[n, n+1)}=$ $\bigcup_{n \in B \cap \mathbf{Z}}[n, n+1]$, and so $\overline{f^{-1}(B)} \backslash f^{-1}(B)=\bigcup_{n \in B \cap \mathbf{Z}}[n, n+1] \backslash \bigcup_{n \in B \cap \mathbf{Z}}[n, n+1) \subseteq$ $\bigcup_{n \in B \cap \mathbf{Z}}([n, n+1] \backslash[n, n+1))=\{n+1: n \in B \cap \mathbf{Z}\} \in \mathcal{I}$.

(3) The function $f:(\mathbf{R}, \mathcal{U}, \mathcal{I}=\mathcal{P}(\{-1,1\})) \rightarrow(\mathbf{R}, \mathcal{U})$ given by $f(x)=-1$ if $x<0$, and $f(x)=1$ if $x \geq 0$, is not $\mathcal{D}$-continuous since if $A=\{-1\}$ then $A=\bar{A}$ and $\overline{f^{-1}(A)} \backslash f^{-1}(\bar{A})=\{0\} \notin \mathcal{I}$. However, $f:(\mathbf{R}, \mathcal{U}) \rightarrow$ $(\mathbf{R}, \mathcal{U}, f(\mathcal{I}))$ is $\mathcal{C}$-continuous given that $f(\mathcal{I})=\mathcal{I}$ and if $A \subseteq \mathbf{R}$ then $f(\bar{A}) \backslash \overline{f(A)} \subseteq\{-1,1\}$ and so $f(\bar{A}) \backslash \overline{f(A)} \in f(\mathcal{I})$.

In the three next theorems we present other characterizations of $\mathcal{D}$ continuity in terms of interior, adherence and frontier operators.

Theorem 5.4 The following propositions are equivalents:

(1) The function $f:(X, \tau, \mathcal{I}) \rightarrow(Y, \beta)$ is $\mathcal{D}$-continuous.

(2) For each $B \subseteq Y, f^{-1}\left(B^{\circ}\right) \backslash$ int $\left(f^{-1}(B)\right) \in \mathcal{I}$.

(3) For each $B \subseteq Y, \overline{f^{-1}(B)} \backslash f^{-1}(\bar{B}) \in \mathcal{I}$.

Proof. It is easy to be established.

Theorem 5.5 The function $f:(X, \tau, \mathcal{I}) \rightarrow(Y, \beta)$ is $\mathcal{D}$-continuous if and only if $\operatorname{Fr}\left(f^{-1}(B)\right) \backslash f^{-1}(F r(B)) \in \mathcal{I}$, for each $B \subseteq Y$.

Proof. $(\rightarrow)$ If $B \subseteq Y$, there exists $\left\{I_{1}, I_{2}\right\} \subseteq \mathcal{I}$ such that $\overline{f^{-1}(B)} \subseteq$ $f^{-1}(\bar{B}) \cup I_{1}$ and $\overline{f^{-1}(Y \backslash B)} \subseteq f^{-1}(\overline{Y \backslash B}) \cup I_{2}$, by Theorem 5.4. Then $\operatorname{Fr}\left(f^{-1}(B)\right)=\overline{f^{-1}(B)} \cap \overline{f^{-1}(Y \backslash B)} \subseteq\left[f^{-1}(\bar{B}) \cup I_{1}\right] \cap\left[f^{-1}(\overline{Y \backslash B}) \cup I_{2}\right]=$ $f^{-1}(F r(B)) \cup I$, where $I=\left[f^{-1}(\bar{B}) \cap I_{2}\right] \cup\left[I_{1} \cap f^{-1}(\overline{Y \backslash B})\right] \cup\left(I_{1} \cap I_{2}\right) \in$ $\mathcal{I}$. Hence $\operatorname{Fr}\left(f^{-1}(B)\right) \backslash f^{-1}(\operatorname{Fr}(B)) \in \mathcal{I}$.

$(\leftarrow)$ If $F \subseteq Y$ is closed, there is a $I \in \mathcal{I}$ such that $\operatorname{Fr}\left(f^{-1}(F)\right) \subseteq$ $f^{-1}(F r(F)) \cup I \subseteq f^{-1}(F) \cup I$, and so $\overline{f^{-1}(F)} \backslash \operatorname{int}\left(f^{-1}(F)\right) \subseteq f^{-1}(F) \cup I$. 
Thus $\overline{f^{-1}(F)} \subseteq \operatorname{int}\left(f^{-1}(F)\right) \cup f^{-1}(F) \cup I=f^{-1}(F) \cup I$. This implies that $\overline{f^{-1}(F)} \backslash f^{-1}(F) \in \mathcal{I}$. By Theorem $5.2 \quad f$ is $\mathcal{D}$-continuous.

Theorem 5.6 The function $f:(X, \tau, \mathcal{I}) \rightarrow(Y, \beta)$ is $\mathcal{D}$-continuous if and only if, for each $A \subseteq X$, there is a $I \in \mathcal{I}$ such that $f(\bar{A} \backslash I) \subseteq \overline{f(A)}$.

Proof. $(\rightarrow)$ If $A \subseteq X$, there is a $I \in \mathcal{I}$ such that $\overline{f^{-1}(f(A))} \backslash f^{-1}(\overline{f(A)})=$ $I$, by Theorem 5.4. Hence $\bar{A} \backslash I \subseteq f^{-1}(\overline{f(A)})$, and so $f(\bar{A} \backslash I) \subseteq \overline{f(A)}$.

$(\leftarrow)$ If $B \subseteq Y$ then there exists $I \in \mathcal{I}$ with $f\left(\overline{f^{-1}(B)} \backslash I\right) \subseteq \overline{f\left(f^{-1}(B)\right)} \subseteq$ $\bar{B}$. This allows us to conclude that $\overline{f^{-1}(B)} \backslash f^{-1}(\bar{B}) \in \mathcal{I}$. By Theorem $5.4, f$ is $\mathcal{D}$-continuous.

Corollary 5.7 If the function $f:(X, \tau, \mathcal{I}) \rightarrow(Y, \beta)$ is $\mathcal{D}$-continuous then $f(\overline{\mathcal{I}}) \subseteq \overline{f(\mathcal{I})}$.

Proof. Let $J \in \overline{\mathcal{I}}$ be. There exists $I \in \mathcal{I}$ such that $J \subseteq \bar{I}$. There is a $I_{1} \in \mathcal{I}$ with $f\left(\bar{I} \backslash I_{1}\right) \subseteq \overline{f(I)}$, by Theorem 5.6. Since we also have that $\underline{f(\bar{I} \backslash)} \backslash f\left(I_{1}\right) \subseteq f\left(\bar{I} \backslash I_{1}\right)$, then $f(J) \subseteq f(\bar{I}) \subseteq \overline{f(I)} \cup f\left(I_{1}\right) \subseteq \overline{f\left(I \cup I_{1}\right)} \in$ $\overline{f(\mathcal{I})}$.

\section{Theorem 5.8}

(1) If $\left\{\mathcal{I}_{\alpha}\right\}_{\alpha \in \Lambda}$ is a collection of ideals in $X$ and if $\mathcal{I}=\bigcap_{\alpha \in \Lambda} \mathcal{I}_{\alpha}$, then $f:(X, \tau, \mathcal{I}) \rightarrow(Y, \beta)$ is $\mathcal{D}$-continuous if and only if

$f:\left(X, \tau, \mathcal{I}_{\alpha}\right) \rightarrow(Y, \beta)$ is $\mathcal{D}$-continuous, for each $\alpha \in \Lambda$.

(2) If $f:(X, \tau, \mathcal{I}) \rightarrow(Y, \beta)$ is $\mathcal{D}$-continuous and $g:(Y, \beta) \rightarrow(Z, \gamma)$ is continuous, then $g \circ f$ is $\mathcal{D}$-continuous.

(3) If $A \subseteq X$ then the function $\chi_{A}:(X, \tau, \mathcal{I}) \rightarrow(\{0,1\}, \mathcal{P}(\{0,1\}))$ is $\mathcal{D}$-continuous if and only if $\operatorname{Fr}(A) \in \mathcal{I}$.

(4) If $A$ and $B$ are open sets in $(X, \tau), X=A \cup B$ and if $f:(X, \tau, \mathcal{I}) \rightarrow$ $(Y, \beta)$ is a function such that the restrictions $f_{A}:\left(A, \tau_{A}, \mathcal{I}_{A}\right) \rightarrow(Y, \beta)$ and $f_{B}:\left(B, \tau_{B}, \mathcal{I}_{B}\right) \rightarrow(Y, \beta)$ are $\mathcal{D}$-continuous, then $f$ is $\mathcal{D}$-continuous.

(5) If $A \subseteq X$ and if $f:(X, \tau, \mathcal{I}) \rightarrow(Y, \beta)$ is $\mathcal{D}$-continuous, then $f_{A}:\left(A, \tau_{A}, \mathcal{I}_{A}\right) \rightarrow(Y, \beta)$ is $\mathcal{D}$-continuous.

Proof. $(3)(\rightarrow)$ Given that $\chi_{A}^{-1}\{0\}$ and $\chi_{A}^{-1}\{1\}$ are open- $\mathcal{I}$, then $\operatorname{Fr}(A)=$ $\bar{A} \backslash A^{\circ}=(\bar{A} \backslash A) \cup\left(A \backslash A^{\circ}\right) \in \mathcal{I}$. $\quad(\leftarrow)$ If $\operatorname{Fr}(A) \in \mathcal{I}$ then $\bar{A} \backslash A \in \mathcal{I}$ and $A \backslash A^{\circ} \in \mathcal{I}$ or, equivalently, $\chi_{A}^{-1}\{0\}$ and $\chi_{A}^{-1}\{1\}$ are open- $\mathcal{I}$. 
(4) Suppose that $V \in \beta$. Given that $\{A, B\} \subseteq \tau$, int $\tau_{\tau_{A}}\left(f_{A}^{-1}(V)\right)=$ $A \cap \operatorname{int}\left(f^{-1}(V)\right)$ and $\operatorname{int}_{\tau_{B}}\left(f_{B}^{-1}(V)\right)=B \cap \operatorname{int}\left(f^{-1}(V)\right)$.

Since $f^{-1}(V)=f_{A}^{-1}(V) \cup f_{B}^{-1}(V)$, we have that $f^{-1}(V) \backslash$ int $\left(f^{-1}(V)\right) \subseteq$ $\left[f_{A}^{-1}(V) \backslash i n t_{\tau_{A}}\left(f_{A}^{-1}(V)\right)\right] \cup\left[f_{B}^{-1}(V) \backslash i n t_{\tau_{B}}\left(f_{B}^{-1}(V)\right)\right] \in \mathcal{I}_{A} \cup \mathcal{I}_{B} \subseteq \mathcal{I}$.

The other parts of the theorem are obtained without any problem.

Theorem 5.9 If $f:(X, \tau, \mathcal{I}) \rightarrow(Y, \beta)$ and $g:(Y, \beta, \mathcal{L}) \rightarrow(Z, \gamma)$ are $\mathcal{D}$ -continuous and if $\left\{f^{-1}(L): L \in \mathcal{L}\right\} \subseteq \mathcal{I}$, then $g \circ f$ is $\mathcal{D}$-continuous.

Proof. If $W \in \gamma$ there exists $L \in \mathcal{L}$ such that $g^{-1}(W)=\operatorname{int}\left(g^{-1}(W)\right) \cup L$. Now, there is a $I \in \mathcal{I}$ with $f^{-1}\left[\operatorname{int}\left(g^{-1}(W)\right)\right]=\operatorname{int}\left\{f^{-1}\left[\operatorname{int}\left(g^{-1}(W)\right)\right]\right\} \cup$ I. So $f^{-1}\left(g^{-1}(W)\right)=f^{-1}\left(\right.$ int $\left.\left(g^{-1}(W)\right)\right) \cup f^{-1}(L)=\operatorname{int}\left\{f^{-1}\left[\right.\right.$ int $\left.\left.\left(g^{-1}(W)\right)\right]\right\} \cup$ $I \cup f^{-1}(L) \subseteq \operatorname{int}\left\{f^{-1}\left(g^{-1}(W)\right)\right\} \cup I \cup f^{-1}(L)$.

Hence $(g \circ f)^{-1}(W) \backslash \operatorname{int}\left[(g \circ f)^{-1}(W)\right] \subseteq I \cup f^{-1}(L) \in \mathcal{I}$.

Corollary 5.10 If $f: X \rightarrow Y$ is one-one and if $g:(Y, \beta, \mathcal{L}) \rightarrow(Z, \gamma)$ and $f:\left(X, \tau, f^{-1}(\mathcal{L})\right) \rightarrow(Y, \beta)$ are $\mathcal{D}$-continuous, then $g \circ f$ is $\mathcal{D}$ -continuous.

The composition of $\mathcal{D}$-continuous functions may not be a $\mathcal{D}$-continuous function, as we show in the following example.

Example 5.11 Suppose that $X=Y=Z=\{a, b, c\}, \tau=\{\emptyset,\{a, b\}, X\}$, $\beta=\{\emptyset,\{a, c\}, Y\}, \gamma=\{\emptyset,\{c\}, Z\}, \mathcal{I}=\mathcal{J}=\mathcal{P}(\{b, c\})$ and that $f: X \rightarrow$ $Y, g: Y \rightarrow Z$ are defined by: $f(a)=b, f(b)=a, f(c)=c, g(a)=b$, $g(b)=c$ and $g(c)=a$. It is not a problem to prove that $f$ and $g$ are $\mathcal{D}$-continuous. Given that $(g \circ f)^{-1}(\{c\}) \backslash \operatorname{int}\left[(g \circ f)^{-1}(\{c\})\right]=\{a\} \notin \mathcal{I}$, we conclude that $g \circ f$ is not $\mathcal{D}$-continuous.

Theorem 5.12 If $\mathcal{I}$ is an ideal in $X$, then $f:\left(X, \tau, \mathcal{I}^{\otimes}\right) \rightarrow(Y, \beta)$ is $\mathcal{D}$ -continuous if and only if $f:(X, \tau \oplus \mathcal{I}) \rightarrow(Y, \beta)$ is continuous.

Proof. $(\rightarrow)$ If $V \in \beta$, there is a $J \in \mathcal{I}^{\otimes}$ such that $f^{-1}(V) \backslash$ int $\left(f^{-1}(V)\right)=$ $J$. So that $f^{-1}(V)=\operatorname{int}\left(f^{-1}(V)\right) \cup J \in \tau \oplus \mathcal{I}$.

$(\leftarrow)$ If $W \in \beta$, there are $U \in \tau$ and $J \in \mathcal{I}^{\otimes}$ such that $f^{-1}(W)=U \cup J$. Then $U \subseteq \operatorname{int}_{\tau}\left(f^{-1}(W)\right)$ and so $f^{-1}(W) \subseteq \operatorname{int}_{\tau}\left(f^{-1}(W)\right) \cup J$. This implies that $f^{-1}(W) \backslash \operatorname{int}_{\tau}\left(f^{-1}(W)\right) \subseteq J$. Thus $f^{-1}(W) \backslash \operatorname{int}_{\tau}\left(f^{-1}(W)\right) \in$ $\mathcal{J}^{\otimes}$. 
It is possible to build new $\mathcal{D}$-continuous functions from some previously known ones, as we will show now.

\section{Theorem 5.13}

(1) If $f:(X, \tau, \mathcal{I}) \rightarrow(Y, \beta)$ and $g:(X, \tau, \mathcal{I}) \rightarrow(Z, \gamma)$ are $\mathcal{D}$-continuous, then the function $h:\left(X, \tau, \mathcal{I}^{\otimes}\right) \rightarrow(Y \times Z, \beta \times \gamma)$, defined by $h(x)=$ $(f(x), g(x))$, is $\mathcal{D}$-continuous.

(2) If $f:(X, \tau, \mathcal{I}) \rightarrow(Y, \beta)$ is $\mathcal{D}$-continuous then the function $g:$ $(X, \tau \oplus \mathcal{I}) \rightarrow(X \times Y,(\tau \oplus \mathcal{I}) \times \beta)$ defined by $g(x)=(x, f(x))$, is continuous. Moreover, if $g$ is continuous then $f:\left(X, \tau, \mathcal{I}^{\otimes}\right) \rightarrow(Y, \beta)$ is $\mathcal{D}$ -continuous.

\section{Proof.}

(1) If $W \in \beta \times \gamma$, there are $\left\{U_{j}: j \in \Lambda\right\} \subseteq \beta$ and $\left\{V_{j}: j \in \Lambda\right\} \subseteq \gamma$ such that $W=\bigcup_{j \in \Lambda}\left(U_{j} \times V_{j}\right)$. Hence $h^{-1}(W)=\bigcup_{j \in \Lambda} h^{-1}\left(U_{j} \times V_{j}\right)=$ $\bigcup_{j \in \Lambda} f^{-1}\left(U_{j}\right) \cap g^{-1}\left(V_{j}\right)$.

For each $j \in \Lambda$ there exists $\left\{I_{j}, J_{j}\right\} \subseteq \mathcal{I}$ such that $f^{-1}\left(U_{j}\right)=i n t\left[f^{-1}\left(U_{j}\right)\right] \cup$ $I_{j}$ and $g^{-1}\left(V_{j}\right)=\operatorname{int}\left[g^{-1}\left(V_{j}\right)\right] \cup J_{j}$.

Thus $h^{-1}(W)=\bigcup_{j \in \Lambda}\left[\operatorname{int}\left(f^{-1}\left(U_{j}\right)\right) \cup I_{j}\right] \cap\left[\operatorname{int}\left(g^{-1}\left(V_{j}\right)\right) \cup J_{j}\right] \subseteq \operatorname{int}\left(h^{-1}(W)\right) \cup$

$I$, where $I=\bigcup_{j \in \Lambda}\left[\operatorname{int}\left(f^{-1}\left(U_{j}\right)\right) \cap J_{j}\right] \cup\left[\operatorname{int}\left(g^{-1}\left(V_{j}\right)\right) \cap I_{j}\right] \cup\left(I_{j} \cap J_{j}\right) \in \mathcal{I}^{\otimes}$.

Hence $h^{-1}(W) \backslash$ int $\left[h^{-1}(W)\right] \in \mathcal{I}^{\otimes}$.

(2) If $U \in \tau$ and $V \in \beta, g^{-1}(U \times V)=U \cap f^{-1}(V) \in \tau \oplus \mathcal{I}$. Now, if $I \in \mathcal{I}$ and $V \in \beta, g^{-1}(I \times V)=I \cap f^{-1}(V) \in \mathcal{I} \subseteq \tau \oplus \mathcal{I}$. This implies that $g$ is continuous.

Finally, if $V \in \beta$ then $f^{-1}(V)=g^{-1}(X \times V) \in \tau \oplus \mathcal{I}$, and so $f^{-1}(V)$ is open- $\mathcal{I}^{\otimes}$.

\section{Theorem 5.14}

(1) If $f:(X, \tau, \mathcal{I}) \rightarrow(Y, \beta)$ is one-one then $f$ is $\mathcal{D}$-continuous if and only if $f:(X, \tau) \rightarrow(Y, \beta, f(\mathcal{I}))$ is $\mathcal{C}$-continuous.

(2) If $f: X \rightarrow Y$ is one-one, then $f:(X, \tau) \rightarrow(Y, \beta, \mathcal{J})$ is $\mathcal{C}$-continuous if and only if $f:\left(X, \tau, f^{-1}(\mathcal{J})\right) \rightarrow(Y, \beta)$ is $\mathcal{D}$-continuous.

Proof. It is easy to be established.

In our final property for this new type of weak continuity, we present the smallest ideal $\mathcal{I}$ in $X$ for which a given function $f:(X, \tau) \rightarrow(Y, \beta)$ is $\mathcal{D}$-continuous. 
Theorem 5.15 If $f:(X, \tau) \rightarrow(Y, \beta)$ is a function and if $\mathcal{I}_{f}=\left\{A \subseteq X:\right.$ there is a finite $\beta_{A} \subseteq \beta$ with $\left.A \subseteq \bigcup_{V \in \beta_{A}}\left[f^{-1}(V) \backslash \operatorname{int}\left(f^{-1}(V)\right)\right]\right\}$,

then $\mathcal{I}_{f}$ is the smallest ideal $\mathcal{I}$ in $X$ such that $f:(X, \tau, \mathcal{I}) \rightarrow(Y, \beta)$ is $\mathcal{D}$-continuous.

Proof. It is clear that $\mathcal{I}_{f}$ is an ideal in $X$. Given that $f^{-1}(V) \backslash \operatorname{int}\left(f^{-1}(V)\right) \in$ $\mathcal{I}_{f}$, for each $V \in \beta$, then $f:\left(X, \tau, \mathcal{I}_{f}\right) \rightarrow(Y, \beta)$ is $\mathcal{D}$-continuous. Now, suppose that $f:(X, \tau, \mathcal{I}) \rightarrow(Y, \beta)$ is $\mathcal{D}$-continuous. If $A \in \mathcal{I}_{f}$ then there is a finite $\beta_{A} \subseteq \beta$ with $A \subseteq \bigcup_{V \in \beta_{A}}\left[f^{-1}(V) \backslash \operatorname{int}\left(f^{-1}(V)\right)\right]$. Since $f^{-1}(V) \backslash \operatorname{int}\left(f^{-1}(V)\right) \in \mathcal{I}$, for each $V \in \beta_{A}$, we can conclude that $A \in \mathcal{I}$. In this way $\mathcal{I}_{f} \subseteq \mathcal{I}$.

In the next example we show that $\mathcal{D}$-continuous and $\mathcal{I}$-continuous (Kaniewski form) are independent concepts.

\section{Example 5.16}

(1) Consider the function $f:(\mathbf{R}, \mathcal{U}, \mathcal{I}=\mathcal{P}(\mathbf{Z})) \rightarrow(\mathbf{R}, \mathcal{L})$ defined by $f(x)=$ $[x]$. Here $\mathcal{L}$ is the Sorgenfrey topology in $\mathbf{R}$. If $V \in \mathcal{L}$ then $f^{-1}(V)=$ $\bigcup_{n \in V \cap \mathbf{Z}}[n, n+1)$ and $\bigcup_{n \in V \cap \mathbf{Z}}(n, n+1) \subseteq \operatorname{int}\left(f^{-1}(V)\right)$. Therefore $f^{-1}(V) \backslash \operatorname{int}\left(f^{-1}(V)\right) \subseteq \mathbf{Z}$. Hence $f^{-1}(V)$ is open- $\mathcal{I}$, and $f$ is $\mathcal{D}$-continuous.

On the other hand, if $V=[0,1)$, we have that $[0,1) \in \mathcal{L}$ but there are no $U \in \mathcal{U}$ and $I \in \mathcal{I}$ such that $f^{-1}(V)=U \backslash I$, because $f^{-1}(V)=[0,1)$. In effect, if $U \in \mathcal{U}, I \in \mathcal{I}$ and $f^{-1}(V)=U \backslash I$, we have that $U=[0,1) \cup(U \cap I)$, and so there exists $\varepsilon \in(0,1)$ such that $(-\varepsilon, 0) \subseteq U \cap I \subseteq \mathbf{Z}$, absurd. In conclusion, $f$ is not $\mathcal{I}$-continuous (Kaniewski form).

(2) Let $\beta=\{\emptyset, \mathbf{R}\} \cup\{(a, \infty): a \in \mathbf{R}\}$ and $g:(\mathbf{R}, \mathcal{U}, \mathcal{I}=\mathcal{P}(\mathbf{R} \backslash \mathbf{Z})) \rightarrow$ $(\mathbf{R}, \beta)$, defined by $g(x)=[x]$. If $r \in \mathbf{R}$ then $g^{-1}((r,+\infty))=[[r]+1,+\infty)=$ $(r,+\infty) \backslash I$, where $I=(r,[r]+1) \in \mathcal{I}$. This implies that $g$ is $\mathcal{I}$-continuous (Kaniewski form).

Now, since $g^{-1}\left(\left(\frac{1}{2},+\infty\right)\right) \backslash \operatorname{int}\left(g^{-1}\left(\left(\frac{1}{2},+\infty\right)\right)\right)=[1,+\infty) \backslash(1,+\infty)=$ $\{1\} \notin \mathcal{I}$, we conclude that $g$ is not $\mathcal{D}$-continuous.

Finally, we show that $\mathcal{D}$-continuous and $\mathcal{I}$-continuous (El Monsef form) are independent concepts.

\section{Example 5.17}

(1) The function $f$ of Example 5.16-(1) is not $\mathcal{I}$-continuous (El Monsef 
form) because $\left(f^{-1}([0,1))\right)^{*}=[0,1)^{*}=[0,1]$ and int $\left[\left(f^{-1}([0,1))\right)^{*}\right]=(0,1)$, and so $f^{-1}([0,1))$ int $\left[\left(f^{-1}([0,1))\right)^{*}\right]$.

(2) If $\lambda=\{\emptyset\} \cup\{A \subseteq \mathbf{R}: \mathbf{R} \backslash A$ is finite $\}$ and $\beta=\{\emptyset, \mathbf{R}\} \cup\{(a, \infty): a \in \mathbf{R}\}$, the function $f:\left(\mathbf{R}, \lambda, \mathcal{I}=\mathcal{I}_{f}(\mathbf{R})\right) \rightarrow(\mathbf{R}, \beta)$ defined by $f(x)=[x]$ is $\mathcal{I}$ continuous (El Monsef form) because

$f^{-1}(\mathbf{R})=\mathbf{R}=\operatorname{int}\left(\mathbf{R}^{*}\right), f^{-1}(\emptyset)=\emptyset=\operatorname{int}\left(\emptyset^{*}\right)$ and, if $a \in \mathbf{R}, f^{-1}((a,+\infty))=$ $[[a]+1,+\infty) \subseteq \operatorname{int}\left[\left(f^{-1}((a,+\infty))\right)^{*}\right]=\mathbf{R}$.

However $f$ is not $\mathcal{D}$-continuous because

$f^{-1}\left(\left(\frac{1}{2},+\infty\right)\right) \backslash \operatorname{int}\left(f^{-1}\left(\left(\frac{1}{2},+\infty\right)\right)\right)=[1,+\infty) \notin \mathcal{I}$.

\section{D-open functions}

In this section we introduce an extension of the open functions to the ideal spaces, in the case in which the considered ideal is defined in the domain of the function.

Definition 6.1 The function $f:(X, \tau, \mathcal{I}) \rightarrow(Y, \beta)$ is said to be $\mathcal{D}$-open if, for each $U \in \tau$, there exists $I \in \mathcal{I}$ such that $f(U \backslash I) \subseteq$ int $(f(U))$.

It is noted that if $f:(X, \tau) \rightarrow(Y, \beta)$ is open then $f:(X, \tau, \mathcal{I}) \rightarrow$ $(Y, \beta) \mathcal{D}$-open, and that if $f:(X, \tau, \mathcal{I}) \rightarrow(Y, \beta)$ is $\mathcal{D}$-open then $f:(X, \tau) \rightarrow$ $(Y, \beta, f(\mathcal{I})) \mathcal{C}$-open. The reciprocal implications, in general, are false, as we can see in the following examples.

\section{Examples 6.2}

1) The function $f:(\mathbf{R}, \mathcal{U}, \mathcal{I}=\mathcal{P}(\mathbf{R})) \rightarrow(\mathbf{R},\{\emptyset, \mathbf{R}\})$, given by $f(x)=x$, is $\mathcal{D}$-open but $f$ is not open.

2) Suppose that $X=Y=\{a, b, c\}, \tau=\{\emptyset,\{a, b\},\{b, c\},\{b\}, X\}, \beta=$ $\{\emptyset,\{a\},\{b\},\{a, b\}, Y\}$ and $\mathcal{I}=\{\emptyset,\{b\}\}$. Let $f: X \rightarrow Y$ be the function defined by $f(a)=b, f(b)=c$ and $f(c)=a$. It is not a problem to prove that $f$ is $\mathcal{D}$-open. Now, given that $f(\{a, b\})=\{b, c\} \notin \beta$ then $f$ is not open.

3) Suppose that $X, Y, \tau$ and $\beta$ are as in example 2, that $\mathcal{I}=\{\emptyset,\{a\}\}$, and that $f: X \rightarrow Y$ is defined by $f(a)=f(b)=c$ and $f(c)=a$. It is observed that $f(\mathcal{I})=\{\emptyset,\{c\}\}$ and that $f:(X, \tau) \rightarrow(Y, \beta, f(\mathcal{I}))$ is $\mathcal{C}$-open. However $f$ is not $\mathcal{D}$-open since there is no a $I \in \mathcal{I}$ such that $f(\{b, c\} \backslash I) \subseteq \operatorname{int}(f(\{b, c\}))$. 


\section{Theorem 6.3}

(1) If $f:(X, \tau, \mathcal{I}) \rightarrow(Y, \beta)$ is $\mathcal{D}$-open and $g:(Y, \beta) \rightarrow(Z, \gamma)$ is open, then $g \circ f$ is $\mathcal{D}$-open.

(2) If $f:(X, \tau, \mathcal{I}) \rightarrow(Y, \beta)$ and $g:(Y, \beta, \mathcal{J}) \rightarrow(Z, \gamma)$ are $\mathcal{D}$-open, $g \circ f$ is one-one and $\mathcal{J} \subseteq f(\mathcal{I})$, then $g \circ f$ is $\mathcal{D}$-open.

\section{Proof.}

1) If $U \in \tau$ then there exists $I \in \mathcal{I}$ such that $f(U \backslash I) \subseteq \operatorname{int}(f(U))$. Hence $f(U) \subseteq \operatorname{int}(f(U)) \cup f(I)$ and $(g \circ f)(U) \subseteq g(\operatorname{int}(f(U))) \cup(g \circ f)(I) \subseteq$ int $[(g \circ f)(U)] \cup(g \circ f)(I)$.

2) If $U \in \tau$ then there exists $I \in \mathcal{I}$ such that $f(U \backslash I) \subseteq \operatorname{int}(f(U))$. Hence $f(U) \subseteq \operatorname{int}(f(U)) \cup f(I)$ and $(g \circ f)(U) \subseteq g(\operatorname{int}(f(U))) \cup(g \circ f)(I)$. Now, there exists $J \in \mathcal{J}$ with $g[\operatorname{int}(f(U)) \backslash J] \subseteq$ int $[g(\operatorname{int}(f(U)))]$. There is a $I_{1} \in \mathcal{I}$ such that $J=f\left(I_{1}\right)$. Thus $g(\operatorname{int}(f(U))) \cup(g \circ f)(I) \subseteq$ $\operatorname{int}[g(\operatorname{int}(f(U)))] \cup(g \circ f)\left(I \cup I_{1}\right) \subseteq \operatorname{int}[(g \circ f)(U)] \cup(g \circ f)\left(I \cup I_{1}\right)$. Since $g \circ f$ is inyective,

$(g \circ f)\left(U \backslash\left(I \cup I_{1}\right)\right)=(g \circ f)(U) \backslash(g \circ f)\left(I \cup I_{1}\right) \subseteq \operatorname{int}[(g \circ f)(U)]$.

The composition of $\mathcal{D}$-open functions may not be a $\mathcal{D}$-open function, as we show in the next example.

Example 6.4 Let $X=Y=Z=\{a, b, c\}, \tau=\{\emptyset, X,\{a, b\},\{b, c\},\{b\}\}$, $\beta=\{\emptyset, Y,\{a\},\{b\},\{a, b\}\}, \gamma=\{\emptyset, Z,\{c\}\}, \mathcal{I}=\{\emptyset,\{b\}\}$ and $\mathcal{J}=\{\emptyset,\{a\}$, $\{b\},\{a, b\}\}$. Consider the functions $f: X \rightarrow Y$ and $g: Y \rightarrow Z$ defined by: $f(a)=b, f(b)=c, f(c)=a$, and $g(a)=b, g(b)=a, g(c)=c$. It is not a problem to verify that $f$ and $g$ are $\mathcal{D}$-open. However, since there is no a $I \in \mathcal{I}$ such that $(g \circ f)[\{a, b\} \backslash I] \subseteq \operatorname{int}[(g \circ f)(\{a, b\})]$, we have that $g \circ f$ is not $\mathcal{D}$-open.

\section{Some applications of $\mathcal{D}$-continuous, $\mathcal{D}$-closed and $\mathcal{D}$-open functions}

In this final section we present some properties of $\mathcal{D}$-continuous and $\mathcal{D}$-open functions, mainly related to compactness and separability. This results also are generalizations of well-known results in general topology.

Recall that an ideal space $(X, \tau, \mathcal{I})$ is: (1) $\rho \mathcal{I}$-compact [9] if, for each collection $\left\{U_{\alpha}\right\}_{\alpha \in \Lambda}$ of open sets, if $X \backslash \bigcup_{\alpha \in \Lambda} U_{\alpha} \in \mathcal{I}$, there exists a finite $\Lambda_{0} \subseteq \Lambda$ such that $X \backslash \bigcup_{\alpha \in \Lambda_{0}} U_{\alpha} \in \mathcal{I}$, and (2) $\sigma \mathcal{I}$-compact [9] if, for each 
non-empty collection $\left\{U_{\alpha}\right\}_{\alpha \in \Lambda}$ of non-empty open sets, if $X \backslash \bigcup_{\alpha \in \Lambda} U_{\alpha} \in \mathcal{I}$, there exists a finite $\Lambda_{0} \subseteq \Lambda$ such that $X=\bigcup_{\alpha \in \Lambda_{0}} U_{\alpha}$.

Theorem 7.1 Suppose that $f:(X, \tau, \mathcal{I}) \rightarrow(Y, \beta)$ is sobreyective and $\mathcal{D}$ -continuous and that $(X, \tau \oplus \mathcal{I})$ is compact. Then $(Y, \beta, f(\mathcal{I}))$ is $f(\mathcal{I})$ -compact.

Proof. If $\left\{V_{\alpha}\right\}_{\alpha \in \Lambda}$ is an open cover of $Y$, then $X=\bigcup_{\alpha \in \Lambda} f^{-1}\left(V_{\alpha}\right)$. For each $\alpha \in \Lambda$ there exists $I_{\alpha} \in \mathcal{I}$ such that $f^{-1}\left(V_{\alpha}\right)=i n t_{\tau}\left(f^{-1}\left(V_{\alpha}\right)\right) \cup I_{\alpha}$. Then $X=\bigcup_{\alpha \in \Lambda}\left(\operatorname{int}_{\tau}\left(f^{-1}\left(V_{\alpha}\right)\right) \cup I_{\alpha}\right)$. Given that $(X, \tau \oplus \tau)$ is compact, there is a finite $\Lambda_{0} \subseteq \Lambda$ such that $X=\bigcup_{\alpha \in \Lambda_{0}}\left(\operatorname{int}_{\tau}\left(f^{-1}\left(V_{\alpha}\right)\right) \cup I_{\alpha}\right)$. Thus $X=\bigcup_{\alpha \in \Lambda_{0}}\left(f^{-1}\left(V_{\alpha}\right) \cup I_{\alpha}\right)$, and this implies $Y=\bigcup_{\alpha \in \Lambda_{0}} V_{\alpha} \cup \bigcup_{\alpha \in \Lambda_{0}} f\left(I_{\alpha}\right)$. Hence $Y \backslash \bigcup_{\alpha \in \Lambda_{0}} V_{\alpha} \subseteq \bigcup_{\alpha \in \Lambda_{0}} f\left(I_{\alpha}\right) \in f(\mathcal{I})$.

Theorem 7.2 Suppose that $f:(X, \tau, \mathcal{I}) \rightarrow(Y, \beta)$ is sobreyective and $\mathcal{D}$ -continuous. Then:

(1) If $\left(X, \tau, \mathcal{I}^{\otimes}\right)$ is $\sigma \mathcal{I}^{\otimes}$-compact then $(Y, \beta)$ is compact.

(2) If $\left(X, \tau, \mathcal{I}^{\otimes}\right)$ is $\rho \mathcal{I}^{\otimes}$-compact then $\left(Y, \beta, f\left(\mathcal{I}^{\otimes}\right)\right)$ is $f\left(\mathcal{I}^{\otimes}\right)$-compact.

(3) If $(X, \tau \oplus \mathcal{I})$ is QHC then $(Y, \beta, f(\overline{\mathcal{I}}))$ is $f(\overline{\mathcal{I}})$-QHC.

\section{Proof.}

(1) If $\left\{V_{\alpha}\right\}_{\alpha \in \Lambda}$ is an open cover of $Y$, then $X=\bigcup_{\alpha \in \Lambda} f^{-1}\left(V_{\alpha}\right)$. For each $\alpha \in \Lambda$ there exists a $I_{\alpha} \in \mathcal{I}$ such that $f^{-1}\left(V_{\alpha}\right)=\operatorname{int}\left(f^{-1}\left(V_{\alpha}\right)\right) \cup I_{\alpha}$. Then $X \backslash \bigcup_{\alpha \in \Lambda} \operatorname{int}\left(f^{-1}\left(V_{\alpha}\right)\right) \subseteq \bigcup_{\alpha \in \Lambda} I_{\alpha} \in \mathcal{I}^{\otimes}$. Since $\left(X, \tau, \mathcal{I}^{\otimes}\right)$ is $\sigma \mathcal{I}^{\otimes}$-compact, there exists a finite $\Lambda_{0} \subseteq \Lambda$ such that $X=\bigcup_{\alpha \in \Lambda_{0}} i n t\left(f^{-1}\left(V_{\alpha}\right)\right)$. Then $X=\bigcup_{\alpha \in \Lambda_{0}} f^{-1}\left(V_{\alpha}\right)$, and so $Y=\bigcup_{\alpha \in \Lambda_{0}} V_{\alpha}$.

(2) If $\left\{V_{\alpha}\right\}_{\alpha \in \Lambda}$ is an open cover of $Y$, then $X=\bigcup_{\alpha \in \Lambda} f^{-1}\left(V_{\alpha}\right)$. For each $\alpha \in \Lambda$ there exists $I_{\alpha} \in \mathcal{I}$ such that $f^{-1}\left(V_{\alpha}\right)=\operatorname{int}\left(f^{-1}\left(V_{\alpha}\right)\right) \cup I_{\alpha}$. Then $X \backslash \bigcup_{\alpha \in \Lambda} \operatorname{int}\left(f^{-1}\left(V_{\alpha}\right)\right) \subseteq \bigcup_{\alpha \in \Lambda} I_{\alpha} \in \mathcal{I}^{\otimes}$. Since $\left(X, \tau, \mathcal{I}^{\otimes}\right)$ is $\rho \mathcal{I}^{\otimes}$-compact, there exists $\Lambda_{0} \subseteq \Lambda$, finite, such that $X \backslash \bigcup_{\alpha \in \Lambda_{0}} i n t\left(f^{-1}\left(V_{\alpha}\right)\right) \in \mathcal{I}^{\otimes}$.

Then $X \backslash \bigcup_{\alpha \in \Lambda_{0}} f^{-1}\left(V_{\alpha}\right) \in \mathcal{I}^{\otimes}$. Given that $Y \backslash \bigcup_{\alpha \in \Lambda_{0}} V_{\alpha} \subseteq f\left(X \backslash \bigcup_{\alpha \in \Lambda_{0}} f^{-1}\left(V_{\alpha}\right)\right) \in$ 
$f\left(\mathcal{I}^{\otimes}\right)$, we have that $Y \backslash \bigcup_{\alpha \in \Lambda_{0}} V_{\alpha} \in f\left(\mathcal{I}^{\otimes}\right)$.

(3) Let $\lambda=\tau \oplus \mathcal{I}$ be. We will denote $a d h_{\tau}(A)$ by $\bar{A}$, for all $A \subseteq X$. If $\left\{V_{\alpha}\right\}_{\alpha \in \Lambda}$ is an open cover of $Y$, then $X=\bigcup_{\alpha \in \Lambda} f^{-1}\left(V_{\alpha}\right)$. For each $\alpha \in \Lambda$ there exists a $I_{\alpha} \in \mathcal{I}$ such that $f^{-1}\left(V_{\alpha}\right)=\operatorname{int}_{\tau}\left(f^{-1}\left(V_{\alpha}\right)\right) \cup I_{\alpha}$. Then $X=\bigcup_{\alpha \in \Lambda}\left[i n t_{\tau}\left(f^{-1}\left(V_{\alpha}\right)\right) \cup I_{\alpha}\right]$.

Since $(X, \tau \oplus \mathcal{I})$ is a $\mathrm{QHC}$ space, there exists a finite $\Lambda_{0} \subseteq \Lambda$ such that $X=\bigcup_{\alpha \in \Lambda_{0}}\left[a d h_{\lambda}\left(\operatorname{int}_{\tau}\left(f^{-1}\left(V_{\alpha}\right)\right)\right) \cup a d h_{\lambda}\left(I_{\alpha}\right)\right] \subseteq \bigcup_{\alpha \in \Lambda_{0}}\left[a d h_{\lambda}\left(f^{-1}\left(V_{\alpha}\right)\right) \cup a d h_{\lambda}\left(I_{\alpha}\right)\right]$.

But $a d h_{\lambda}(A) \subseteq \bar{A}$, for each $A \subseteq X$.

Then $X=\bigcup_{\alpha \in \Lambda_{0}}\left[\overline{f^{-1}\left(V_{\alpha}\right)} \cup \overline{I_{\alpha}}\right]$, and so $Y=\bigcup_{\alpha \in \Lambda_{0}} f\left[\overline{f^{-1}\left(V_{\alpha}\right)}\right] \cup \bigcup_{\alpha \in \Lambda_{0}} f\left(\overline{I_{\alpha}}\right)$.

For each $\alpha \in \Lambda_{0}$ there exists $J_{\alpha} \in \mathcal{I}$ with $\frac{\alpha \in \Lambda_{0}}{f^{-1}\left(V_{\alpha}\right)} \backslash f^{-1}\left(a d h_{\beta}\left(V_{\alpha}\right)\right)=$ $J_{\alpha}$. Hence $Y=\bigcup_{\alpha \in \Lambda_{0}} f\left(\overline{I_{\alpha}}\right) \cup \bigcup_{\alpha \in \Lambda_{0}} f\left[f^{-1}\left(a d h_{\beta}\left(V_{\alpha}\right)\right)\right] \cup \bigcup_{\alpha \in \Lambda_{0}} f\left(J_{\alpha}\right)=$ $\bigcup_{\alpha \in \Lambda_{0}} f\left(\overline{I_{\alpha}}\right) \cup \bigcup_{\alpha \in \Lambda_{0}} a d h_{\beta}\left(V_{\alpha}\right) \cup \bigcup_{\alpha \in \Lambda_{0}} f\left(J_{\alpha}\right)$. Consecuently $Y \backslash \bigcup_{\alpha \in \Lambda_{0}} a d h_{\beta}\left(V_{\alpha}\right) \in$ $f(\overline{\mathcal{I}})$.

Now we present some properties of the $\mathcal{D}$-continuous functions, in the case in which the codomain is a Hausdorf space.

Theorem 7.3

(1) If $f:(X, \tau, \mathcal{I}) \rightarrow(Y, \beta)$ and $g:(X, \tau, \mathcal{I}) \rightarrow(Y, \beta)$ are $\mathcal{D}$-continuous functions, and if $(Y, \beta)$ is $\mathrm{T}_{2}$, then $A=\{x \in X: f(x)=g(x)\}$ is closed in $(X, \tau \oplus \mathcal{I})$.

(2) If $f:(X, \tau, \mathcal{I}) \rightarrow(Y, \beta)$ is $\mathcal{D}$-continuous and $(Y, \beta)$ is $\mathrm{T}_{2}$, then $A=$ $\{(u, v) \in X \times X: f(u)=f(v)\}$ is closed in $(X \times X,(\tau \oplus \mathcal{I}) \times(\tau \oplus \mathcal{I}))$.

\section{Proof.}

(1) Suppose that $u \in X \backslash A$. There is a $\{U, V\} \subseteq \beta$ with $f(u) \in U, g(u) \in V$ and $U \cap V=\emptyset$. Then $u \in f^{-1}(U) \cap g^{-1}(V), A \cap\left[f^{-1}(U) \cap g^{-1}(V)\right]=\emptyset$ and $f^{-1}(U) \cap g^{-1}(V) \in \tau \oplus \mathcal{I}$, because $f^{-1}(U) \cap g^{-1}(V)$ is open- $\mathcal{I}$.

(2) Suppose that $(u, v) \in(X \times X) \backslash A$. There is a $\{U, V\} \subseteq \beta$ with $f(u) \in$ $U, f(v) \in V$ and $U \cap V=\emptyset$. Then $(u, v) \in f^{-1}(U) \times f^{-1}(V), A \cap$ $\left[f^{-1}(U) \times g^{-1}(V)\right]=\emptyset$ and $f^{-1}(U) \times f^{-1}(V) \in(\tau \oplus \mathcal{I}) \times(\tau \oplus \mathcal{I})$, because $f^{-1}(U)$ and $f^{-1}(V)$ are open- $\mathcal{I}$. This allows us to conclude that $A$ is closed in the space $(X \times X,(\tau \oplus \mathcal{I}) \times(\tau \oplus \mathcal{I}))$.

Recall that an ideal space $(X, \tau, \mathcal{I})$ is said to be $\mathcal{J}$-Hausdorff [13] if for 
each $\{a, b\} \subseteq X$ with $a=b$, there exists $\{U, V\} \subseteq \tau$ such that $a \in U, b \in V$ and $U \cap V \in \mathcal{I}$.

Theorem 7.4 If $f:(X, \tau, \mathcal{I}) \rightarrow(Y, \beta)$ is biyective and $\mathcal{D}$-open, and if $(X, \tau, \mathcal{I})$ is $\mathcal{J}$-Hausdorff, then $(Y, \beta \oplus f(\mathcal{I}), f(\mathcal{I}))$ is $\mathcal{J}$-Hausdorff.

Proof. Suppose that $\{a, b\} \subseteq X$ and that $f(a)=f(b)$. There exists $\{U, V\} \subseteq \tau$ such that $a \in U, b \in V$ and $U \cap V \in \mathcal{I}$. Given that int $(f(U)) \cap$ int $(f(V)) \subseteq f(U) \cap f(V)=f(U \cap V) \in f(\mathcal{I})$, we have that int $(f(U)) \cap$ int $(f(V)) \in f(\mathcal{I})$. On the other hand, there exists $\left\{I_{1}, I_{2}\right\} \subseteq \mathcal{I}$ such that $f\left(U \backslash I_{1}\right) \subseteq \operatorname{int}(f(U))$ and $f\left(V \backslash I_{2}\right) \subseteq \operatorname{int}(f(V))$.

Hence:

(i) $f(a) \in \operatorname{int}(f(U)) \cup f\left(I_{1}\right), f(b) \in \operatorname{int}(f(V)) \cup f\left(I_{2}\right)$,

(ii) $\left\{\right.$ int $(f(U)) \cup f\left(I_{1}\right)$, int $\left.(f(V)) \cup f\left(I_{2}\right)\right\} \subseteq \beta \oplus f(\mathcal{I})$ and $($ iii $)\left[\right.$ int $\left.(f(U)) \cup f\left(I_{1}\right)\right] \cap$ $\left[\operatorname{int}(f(V)) \cup f\left(I_{2}\right)\right]=[\operatorname{int}(f(U)) \cap \operatorname{int}(f(V))] \cup\left[f\left(I_{1}\right) \cap \operatorname{int}(f(V))\right] \cup\left[\operatorname{int}(f(U)) \cap f\left(I_{2}\right)\right] \cup$ $\left[f\left(I_{1}\right) \cap f\left(I_{2}\right)\right] \in f(\mathcal{I})$.

\section{References}

[1] M. E. Abd El-Monsef, E. F. Lashien, and A.A. Nasef, "On I-open sets and I-continuous functions", Kyungpook mathematical journal, vol. 32, no. 1, pp. 21-30, Jun. 1992. [On line]. Available: https:// bit.ly/3mP7S71

[2] A. Çobankaya, F. Kuyucu, and S. Kilinç, "New approaches about I-continuous functions in ideal topological spaces", International journal of pure and applied mathematics, vol.113, no. 2, pp. 291-297, 2017. [On line]. Available: https:// bit.ly/2FNfmqs

[3] M. K. Gupta and T. Noiri, "C-compactness modulo an ideal", International journal of mathematics and mathematical sciences, Art ID. 078135, 2006, doi: 10.1155/ IJMMS/ 2006/78135

[4] D. Jancovic and T.R. Hamlett, "Compatible extensions of ideals", Unione Matematica Italiana Bollettino. B. Serie 7, vol. 6, no. 3, pp. 453-465, 1992

[5] D. Jancovic and T.R. Hamlett, "New topologies from old via ideals", The american mathematical monthly, vol. 97, no. 4, pp. 295-310, Apr. 1990, doi: 10.1080/ 00029890.1990.11995593

[6] J. Kaniewski and Z. Piotrowski, "Concerning continuity apart from a meager set", Proceedings of the American Mathematical Society, vol. 98, no. 2, pp. 324-328, 1986, doi: 10.1090/S0002-9939-19860854041-3 
[7] R. Newcomb, "Topologies which are compact modulo an ideal", Ph.D. dissertation, University of California at Santa Barbara, 1967.

[8] A. Özkurt, "Some generalizations of local continuity in ideal topological spaces", Scientific Studies and Research. Series Mathematics and Informatics (Online), vol. 24, no. 1, pp. 75-80, 2014. [On line]. Available: https:// bit.ly/3hSC7pS

[9] N. R. Pachón Rubiano, "New forms of strong compactness in terms of ideals", International journal of pure and applied mathematics, vol. 106, no. 2, pp. 481-493, 2016, doi: 10.12732/ijpam.v106i2.12

[10] N. R. Pachón Rubiano,"Between closed and Ig-closed sets", European journal of pure and applied mathematics, vol. 11, no. 1, p. 299, Jan. 2018, doi: 10.29020/ nybg.ejpam.v11i2.3131

[11] J. Porter and J. Thomas, "On H-closed and minimal Hausdorff spaces", Transactions of the American Mathematical Society, vol. 138, pp. 159-170, 1969, doi: 10.1090/S0002-9947-1969-0238268-4

[12] V. Renukadevi and D. Sivaraj, "A generalization of normal spaces", Archivum mathematicum, vol. 44, no. 4, pp. 265-270, 2008. [On line]. Avaiable: https:/ / bit.ly/2FSj9To

[13] S. Suriyakala and R. Vembu, "On separation axioms in ideal topological spaces", Malaya journal of matematik, vol. 4, no. 2, pp. 318-324, 2016. [On line]. Available: https:// bit.ly/3iWOdji 